CRYSTALLOGRAPHIC COMMUNICATIONS

ISSN 2056-9890

Received 7 October 2016

Accepted 20 October 2016

Edited by P. C. Healy, Griffith University, Australia

Keywords: crystal structure; carbohydrazide; methylation; weak hydrogen bonds.

CCDC references: $1510866 ; 1510865$; 1510864

Supporting information: this article has supporting information at journals.iucr.org/e

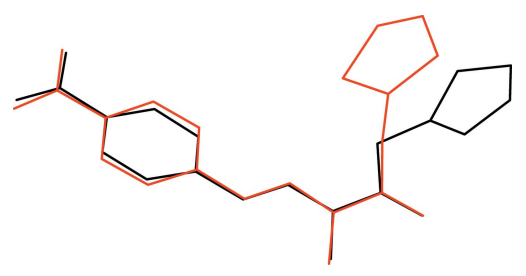

OPEN $\odot$ ACCESS

\section{Different weak interactions in the crystals of three isomeric $(E)-N$-methyl- $N^{\prime}$-(nitrobenzylidene)- 2-(thiophen-2-yl)acetohydrazides}

\author{
Laura N. F. Cardoso, ${ }^{a, b}$ Thais C. M. Noguiera, ${ }^{a}$ Carlos R. Kaiser, ${ }^{b}$ James L. \\ Wardell, ${ }^{a, c}$ Marcus V. N. de Souza, ${ }^{a}$ Shaun T. Lancaster ${ }^{c}$ and William T. A. \\ Harrison $^{\mathrm{c} *}$
}

\begin{abstract}
${ }^{\text {a}}$ Fundação Oswaldo Cruz, Instituto de Tecnologia em Fármacos-FarManguinhos, Rua Sizenando Nabuco, 100, Manguinhos, 21041-250 Rio de Janeiro, Brazil, 'b Instituto de Química, Universidade Federal do Rio de Janeiro, Cidade Universitária, Rio de Janeiro, Brazil, and ${ }^{\mathrm{c}}$ Department of Chemistry, University of Aberdeen, Meston Walk, Aberdeen AB24 3UE, Scotland. *Correspondence e-mail: w.harrison@abdn.ac.uk
\end{abstract}

The crystal structures of three isomeric $(E)-N$-methyl- $N^{\prime}$-(nitrobenzylidene)-2(thiophen-2-yl)acetohydrazides (formula $\mathrm{C}_{14} \mathrm{H}_{13} \mathrm{~N}_{3} \mathrm{O}_{3} \mathrm{~S}$ ) are described, with the nitro group in ortho, meta and para positions in the benzene ring. In each crystal structure, molecules are linked by various weak interactions $(\mathrm{C}-\mathrm{H} \cdots \mathrm{O}$ and $\mathrm{C}-\mathrm{H} \cdots \pi$ bonds, and $\pi-\pi$ stacking), leading to three-dimensional networks in each case, but with little similarity between them.

\section{Chemical context}

Our ongoing interest in the biological activities and structural chemistry of heterocyclic compounds have led us to investigate compounds containing a thiophene ring system. We have reported the syntheses and anti-TB activities of acetamido derivatives, 2- $\left(R, R^{\prime} \mathrm{NCOCH}_{2}\right)$-thiophene (de Souza et al., $2008)$ and more recently thienyl acetohydrazide derivatives, 2- $\left(\mathrm{ArCH}=\mathrm{N}-\mathrm{NHCOCH}_{2}\right)$-thiophene (Cardoso et al., 2014). We have followed up this study with work on $(E)-N$-methyl$N^{\prime}$-arylidene-2-(thiophen-2-yl)acetohydrazides. The anti-TB activities of these compounds will be reported elsewhere: here, we present the crystal structures of three isomeric derivatives in this family bearing a nitro group on the aromatic ring, viz. (E)-N-methyl- $N^{\prime}$-(2-nitronitrobenzylidene)-2-(thiophen2-yl)acetohydrazide, (I), (E)- $N$-methyl- $N^{\prime}$-(3-nitronitrobenzylidene)-2-(thiophen-2-yl)acetohydrazide, (II), and (E)-Nmethyl- $N^{\prime}$-(4-nitronitrobenzylidene)-2-(thiophen-2-yl)acetohydrazide, (III).

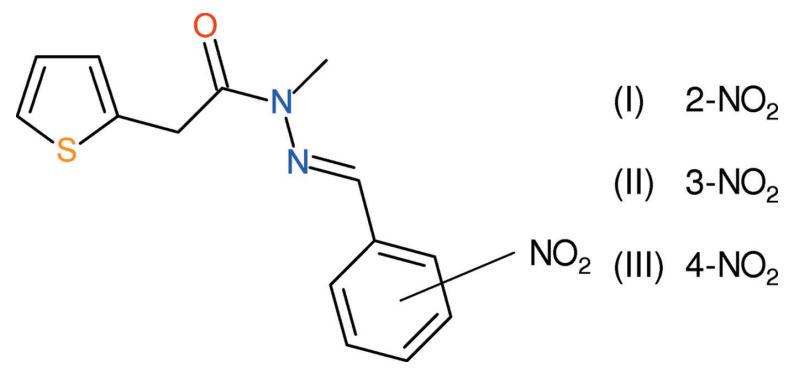

\section{Structural commentary}

The molecular structure of (I) is shown in Fig. 1, which confirms that methylation has occurred at N2. The thiophene 


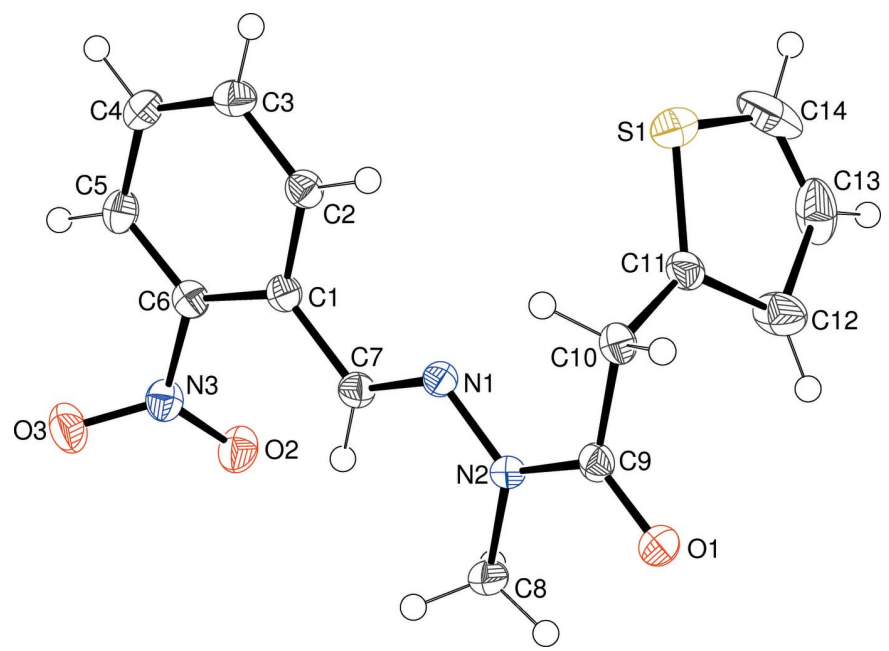

Figure 1

The molecular structure of (I), showing 50\% displacement ellipsoids. Only the major orientation of the thiophene ring is shown.

ring (S1/C11-C14) shows 'flip' disorder (compare, for example, Sonar et al., 2005; Wagner et al., 2006) over two conformations rotated by $\sim 180^{\circ}$ about the $\mathrm{C} 10-\mathrm{C} 11$ bond in a 0.671 (2):0.329 (2) ratio. The dihedral angle between the thiophene ring and the $\mathrm{C} 1-\mathrm{C} 6$ benzene ring is $77.22(6)^{\circ}$. The ortho-N3/O1/O2 nitro group deviates from the mean plane of its attached benzene ring by $43.61(5)^{\circ}$ : this substantial twist can in part be ascribed to steric reasons. The central $\mathrm{CH}=\mathrm{N}-$ $\mathrm{N}\left(\mathrm{CH}_{3}\right)-\mathrm{C}(=\mathrm{O})-\mathrm{CH}_{2}$ fragment in (I) is approximately planar (r.m.s. deviation $=0.032 \AA$ ) and subtends dihedral angles of $6.39(5)$ and $83.61(6)^{\circ}$ with the benzene and thiophene rings, respectively. Thus, the major twist in the molecule occurs about the $\mathrm{C} 9-\mathrm{C} 10$ bond $[\mathrm{N} 2-\mathrm{C} 9-\mathrm{C} 10-\mathrm{C} 11=$ $-81.73(18)^{\circ}$ ], giving the molecule an approximate overall Lshape. The $\mathrm{N} 1-\mathrm{N} 2$ bond length of $1.3725(18)^{\circ}$ is shorter than the reference value of $\sim 1.41 \AA$ for an $\mathrm{N}-\mathrm{N}$ single bond and

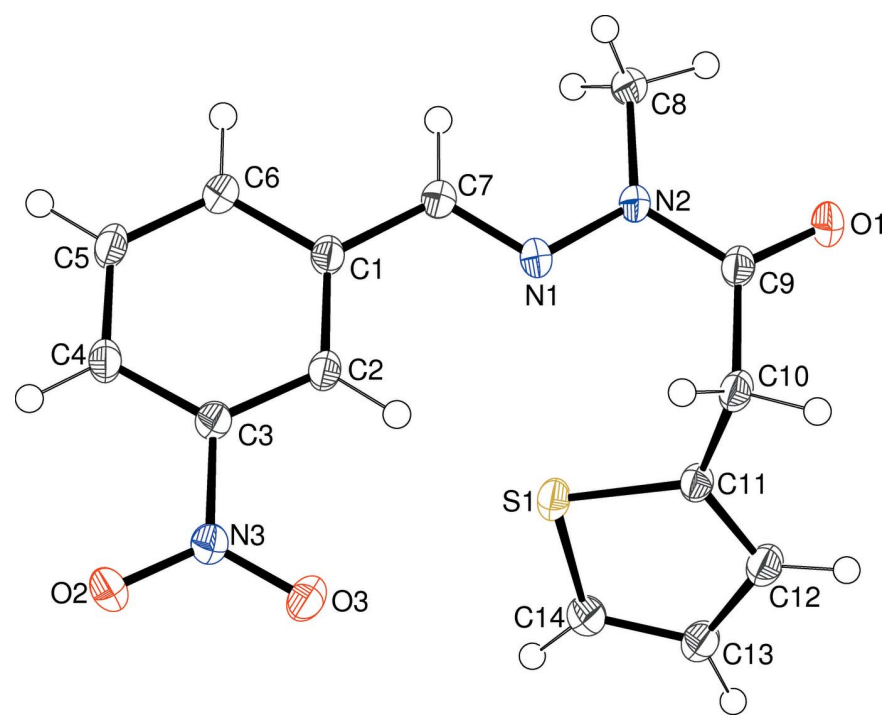

Figure 2

The molecular structure of (II), showing $50 \%$ displacement ellipsoids.

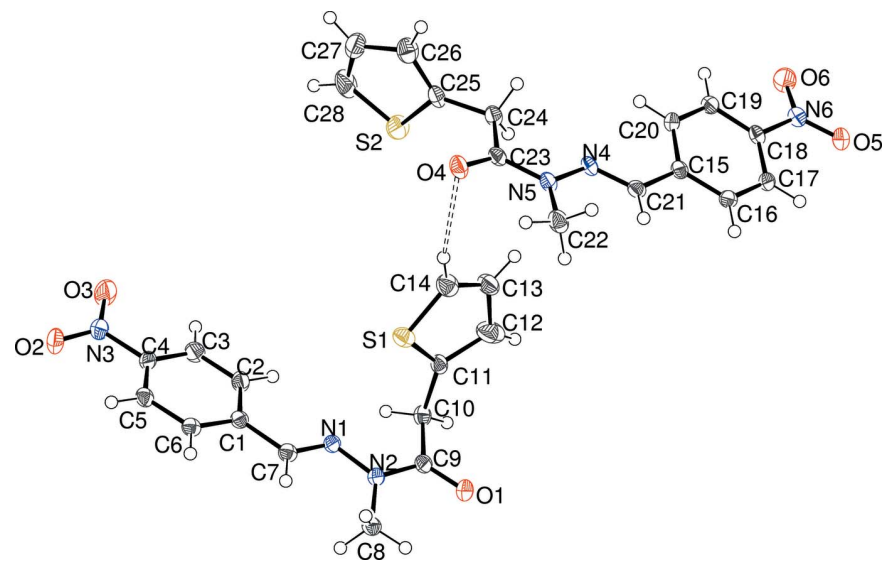

Figure 3

The molecular structure of (III), showing 50\% displacement ellipsoids. Only the major orientation of the thiophene ring is shown.

the $\mathrm{C} 9-\mathrm{N} 2$ amide bond of 1.377 (2) $\AA$ is somewhat lengthened: these distance data suggest significant delocalization of electrons over the methylidene-acetohydrazide grouping.

The molecular structure of (II) can be seen in Fig. 2; again the methylation of $\mathrm{N} 2$ has occurred as expected but this time the S1/C11-C14 thiophene ring shows no detectable sign of disorder $\left[\mathrm{C} 11-\mathrm{S} 1-\mathrm{C} 14=92.35(6)^{\circ}\right]$. The dihedral angle between the thiophene ring and the $\mathrm{C} 1-\mathrm{C} 6$ benzene ring is $60.17(4)^{\circ}$. The meta-N3/O1/O2 nitro group is almost coplanar with its attached benzene ring [dihedral angle $=1.96(2)^{\circ}$ ]. The almost planar central methylidene-acetohydrazide grouping in (II) (r.m.s. deviation $=0.006 \AA$ ) subtends dihedral angles of $7.27(7)^{\circ}$ with the benzene ring and $61.67(4)^{\circ}$ with the thiophene ring. As in (I), the major twist occurs about $\mathrm{C} 9-\mathrm{C} 10$ $\left[\mathrm{N} 2-\mathrm{C} 9-\mathrm{C} 10-\mathrm{C} 11=85.18(14)^{\circ}\right]$, again giving the molecule an approximate overall L-shape. The $\mathrm{N} 1-\mathrm{N} 2$ and $\mathrm{C} 9-\mathrm{N} 2$ bond lengths in (II) are 1.3747 (14) and 1.3776 (15) $\AA$, respectively, which again can be ascribed to delocalization.

Compound (III) crystallizes with two molecules (methylated at N2 and N5) in the asymmetric unit with different conformations (Fig. 3); in both molecules the thiophene ring is rotationally disordered [major/minor disorder components = 0.673 (3):0.327 (3) for the S1 ring and 0.832 (3):0.168 (3) for the $\mathrm{S} 2$ ring. In the $\mathrm{S} 1$ molecule, the dihedral angles between the benzene ring ' $A$ ', thiophene ring ' $B$ ' and $\mathrm{CH}=\mathrm{N}-$

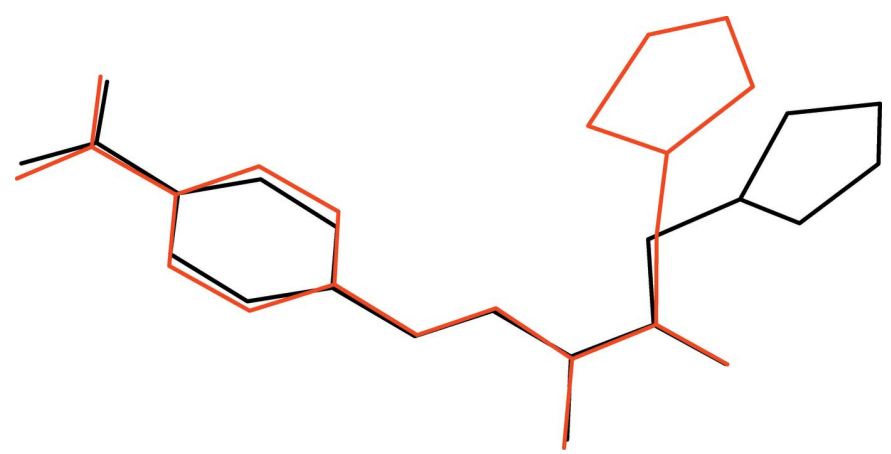

Figure 4

Overlay plot of the N1 (red) and N4 (black) molecules in (III). 


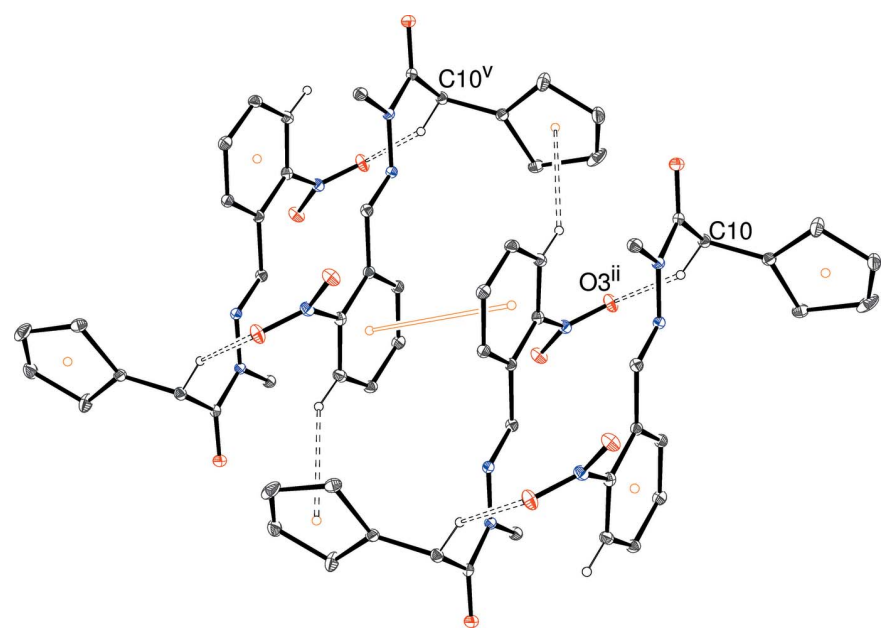

Figure 5

Fragment of a [100] hydrogen-bonded chain in the crystal of (I). [Symmetry codes: (ii) $-x, 1-y, 1-z$; (v) $1+x, y, z$.] All $\mathrm{H}$ atoms not involved in hydrogen bonds have been omitted for clarity.

$\mathrm{N}\left(\mathrm{CH}_{3}\right)-\mathrm{C}(=\mathrm{O})-\mathrm{CH}_{2}$ fragment ' $C$ ' (r.m.s. deviation = $0.034 \AA$ ), are $A / B=79.36(6), A / C=12.75(12)$ and $B / C=$ $69.60(6)^{\circ}$. Equivalent dihedral-angle data for the S2 molecule are $88.23(6), 15.51(13)$ and $82.51(6)^{\circ}$, respectively. The paranitro group is twisted from its attached ring by 9.2 (3) (S1 molecule) and $8.8(3)^{\circ}$ (S2 molecule). The dihedral angles are broadly similar but even so, the two molecules have different conformations (Fig. 4) as indicated by the N2-C9-C10$\mathrm{C} 11$ and $\mathrm{N} 5-\mathrm{C} 23-\mathrm{C} 24-\mathrm{C} 25$ torsion angles of 91.7 (2) and $171.09(17)$, respectively. Bond-length data $[\mathrm{N} 1-\mathrm{N} 2=$ 1.373 (2), $\mathrm{C} 9-\mathrm{N} 2=1.380(3), \mathrm{N} 4-\mathrm{N} 5=1.368$ (2) and C23$\mathrm{N} 5=1.384(2) \AA]$ are consistent between the molecules and with the equivalent data for (I) and (II).

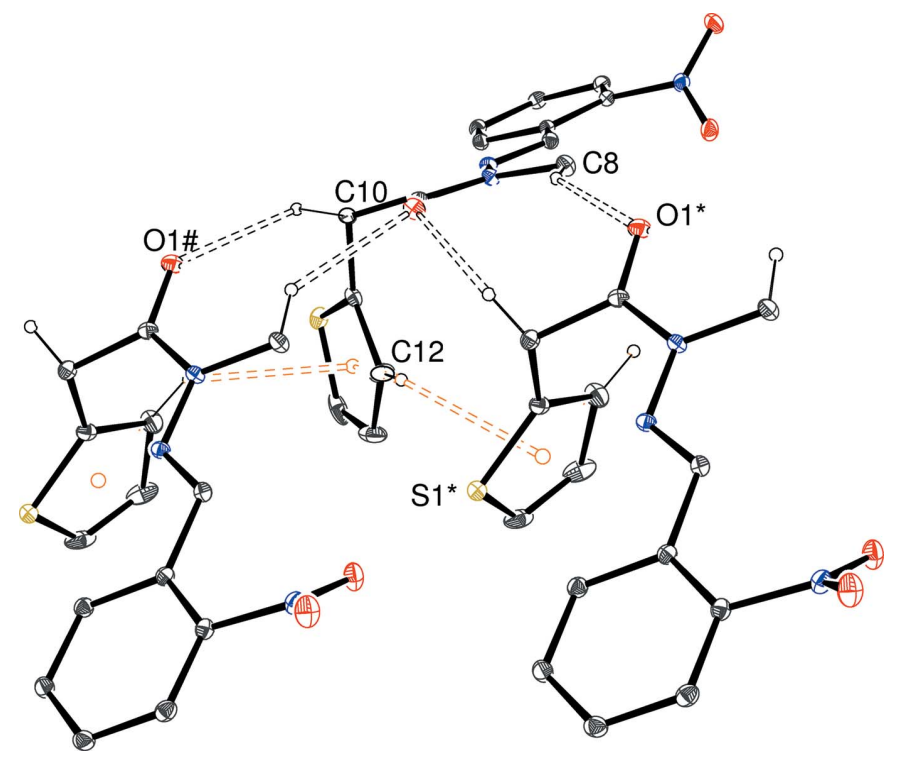

Figure 6

Fragment of an [001] hydrogen-bonded chain in the crystal of (I). [Symmetry codes: (*) $x, \frac{1}{2}-y, z-\frac{1}{2}$; (\#) $x, \frac{1}{2}-y, \frac{1}{2}+z$.] All $\mathrm{H}$ atoms not involved in hydrogen bonds have been omitted for clarity.
Table 1

Hydrogen-bond geometry $\left(\AA,^{\circ}\right)$ for (I).

$C g 1$ is the centroid of the thiophene ring.

\begin{tabular}{lllll}
\hline$D-\mathrm{H} \cdots A$ & $D-\mathrm{H}$ & $\mathrm{H} \cdots A$ & $D \cdots A$ & $D-\mathrm{H} \cdots A$ \\
\hline $\mathrm{C} 8-\mathrm{H} 8 C \cdots \mathrm{O} 1^{\mathrm{i}}$ & 0.98 & 2.49 & $3.293(2)$ & 139 \\
$\mathrm{C} 10-\mathrm{H} 10 A \cdots \mathrm{O} 3^{\mathrm{ii}}$ & 0.99 & 2.55 & $3.386(2)$ & 142 \\
$\mathrm{C} 10-\mathrm{H} 10 B \cdots \mathrm{O} 1^{\mathrm{iii}}$ & 0.99 & 2.52 & $3.439(2)$ & 154 \\
$\mathrm{C} 5-\mathrm{H} 5 \cdots C g 1^{\mathrm{iv}}$ & 0.95 & 2.86 & $3.7212(18)$ & 151 \\
$\mathrm{C} 12-\mathrm{H} 12 \cdots C g 1^{\mathrm{i}}$ & 0.95 & 2.85 & $3.5930(13)$ & 136 \\
\hline
\end{tabular}

Symmetry codes: (i) $x,-y+\frac{1}{2}, z-\frac{1}{2}$; (ii) $-x,-y+1,-z+1$; (iii) $x,-y+\frac{1}{2}, z+\frac{1}{2}$; (iv) $-x+1,-y+1,-z+1$.

\section{Supramolecular features}

The packing in (I) can be decomposed into two different chains: in the first of these (Fig. 5), inversion dimers (about the point $0, \frac{1}{2}, \frac{1}{2}$ for the asymmetric molecule) linked by pairs of C10-H10a. . O3 hydrogen bonds (Table 1) generate $R_{2}^{2}(20)$ loops. These dimers are complemented by inversion-related pairs of $\mathrm{C} 5-\mathrm{H} 5 \cdots \mathrm{Cg} 1$ (where $\mathrm{Cg} 1$ is the centroid of the thiophene ring) bonds; this second inversion dimer (about $\frac{1}{2}, \frac{1}{2}$, $\frac{1}{2}$ ) is reinforced by an aromatic $\pi-\pi$ stacking interaction involving the $\mathrm{C} 1-\mathrm{C} 6$ benzene rings [centroid separation = 3.7118 (9) $\AA$; slippage $=1.27 \AA$. Together, the $\mathrm{C}-\mathrm{H} \cdots \mathrm{O}$ dimers and the $\mathrm{C}-\mathrm{H} \cdots \pi+\pi-\pi$ dimers alternate in [100] chains. In the second one-dimensional motif, the $\mathrm{C} 8, \mathrm{C} 10-$ $\mathrm{H} 10 \mathrm{~b}$ and $\mathrm{C} 12$ bonds combine together to generate [001] chains (Fig. 6) in which the carbonyl O1 atom accepts hydrogen bonds from two adjacent molecules to generate $R_{2}^{2}(9)$ loops. The cohesion of the chain is reinforced by a C$\mathrm{H} \cdots \pi$ interaction from one thiophine ring to the next: the dihedral angle between two adjacent rings in the chain is $73.32(4)^{\circ}$. Taken together, the [100] and [001] chains combine together to generate a three-dimensional network.

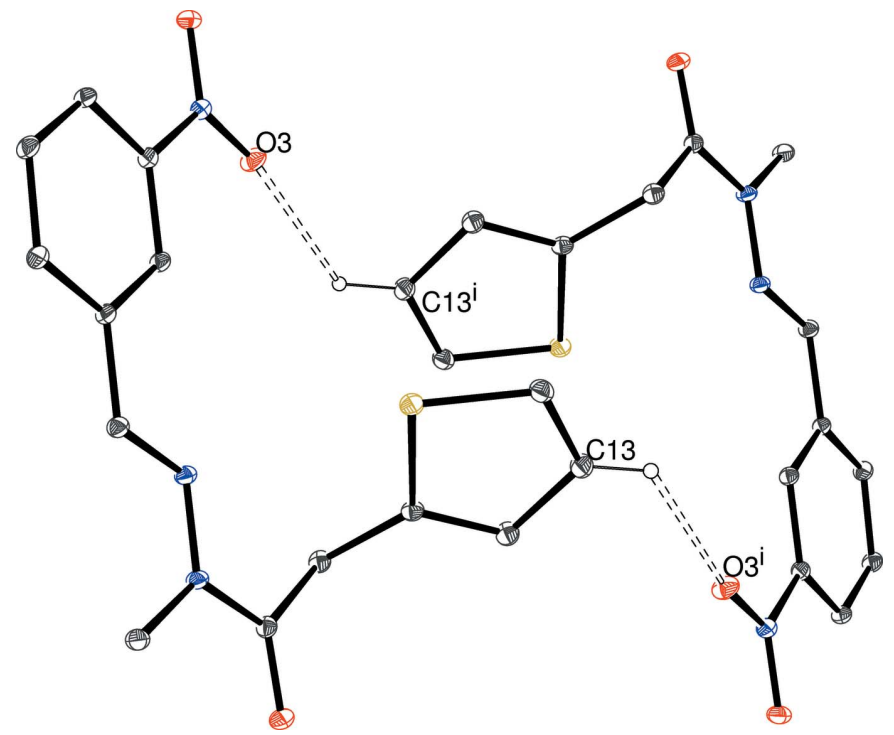

Figure 7

Inversion dimer in the crystal of (II) linked by a pair of $\mathrm{C}-\mathrm{H} \cdots \mathrm{O}$ hydrogen bonds. [Symmetry code: (i) $2-x, 1-y, 1-z$.] All $\mathrm{H}$ atoms not involved in hydrogen bonds have been omitted for clarity. 
Table 2

Hydrogen-bond geometry $\left(\AA,^{\circ}\right)$ for (II).

\begin{tabular}{lllll}
\hline$D-\mathrm{H} \cdots A$ & $D-\mathrm{H}$ & $\mathrm{H} \cdots A$ & $D \cdots A$ & $D-\mathrm{H} \cdots A$ \\
\hline $\mathrm{C} 7-\mathrm{H} 7 \cdots \mathrm{O} 2^{\mathrm{i}}$ & 0.95 & 2.39 & $3.2879(16)$ & 157 \\
$\mathrm{C} 8-\mathrm{H} 8 B \cdots \mathrm{O} 2^{\text {ii }}$ & 0.98 & 2.50 & $3.3468(16)$ & 144 \\
$\mathrm{C} 8-\mathrm{H} 8 C \cdots 3^{\text {iii }}$ & 0.98 & 2.52 & $3.4356(17)$ & 156 \\
$\mathrm{C}^{2}-\mathrm{H} 13 \cdots 3^{\text {iv }}$ & 0.95 & 2.52 & $3.1874(16)$ & 127 \\
\hline
\end{tabular}

Symmetry codes: (i) $-x+\frac{1}{2}, y-\frac{1}{2},-z+\frac{1}{2}$; $-x+\frac{3}{2}, y-\frac{1}{2},-z+\frac{1}{2}$; (iv) $-x+2,-y+1,-z+1$.

(ii) $\quad x+\frac{1}{2},-y+\frac{1}{2}, z+\frac{1}{2}$

The packing in (II) features four $\mathrm{C}-\mathrm{H} \cdots \mathrm{O}$ interactions (Fig. 7, Table 2); the $\mathrm{C} 13$ bond (Fig. 2) generates $R_{2}^{2}(28)$ loops and the $C 7$ bond leads to $C(7)$ chains propagating in [010]. The two C8 (methyl-group) bonds lead to (101) sheets. Taken together, these interactions lead to a three-dimensional network of molecules in the crystal. There are no $\mathrm{C}-\mathrm{H} \cdots \pi$ or $\pi-\pi$ stacking interactions in (II).

The packing for (III) can be visualized in terms of two different chains. The first of these (Table 3, Figs. 8 and 9), which involves the four $\mathrm{C}-\mathrm{H}$ donor groups of the $\mathrm{C} 1-\mathrm{mol}-$ ecule, is built up from inversion dimers (about the point 1,0,0 for the asymmetric molecule) of C1-molecules linked by pairs of $\mathrm{C} 5-\mathrm{H} 5 \cdots \mathrm{O} 2$ hydrogen bonds, which generate $R_{2}^{2}(8)$ loops. The $\mathrm{C} 6-\mathrm{H} 6$ and $\mathrm{C} 7-\mathrm{H} 7$ groups link to the same acceptor atom (O6; part of the $\mathrm{C} 15$ molecule), to generate an $R_{2}^{1}(6)$
Table 3

Hydrogen-bond geometry $\left(\AA,^{\circ}\right)$ for (III).

Cg6 is the centroid of the $\mathrm{C} 15-\mathrm{C} 20$ ring.

\begin{tabular}{lllll}
\hline$D-\mathrm{H} \cdots A$ & $D-\mathrm{H}$ & $\mathrm{H} \cdots A$ & $D \cdots A$ & $D-\mathrm{H} \cdots A$ \\
\hline $\mathrm{C} 5-\mathrm{H} 5 \cdots \mathrm{O} 2^{\mathrm{i}}$ & 0.95 & 2.48 & $3.312(3)$ & 147 \\
$\mathrm{C} 6-\mathrm{H} 6 \cdots 6^{\mathrm{ii}}$ & 0.95 & 2.56 & $3.412(2)$ & 149 \\
$\mathrm{C} 7-\mathrm{H} 7 \cdots 6^{\mathrm{ii}}$ & 0.95 & 2.41 & $3.281(3)$ & 153 \\
$\mathrm{C} 14-\mathrm{H} 14 \cdots \mathrm{O} 4$ & 0.95 & 2.55 & $3.464(3)$ & 160 \\
$\mathrm{C} 17-\mathrm{H} 17 \cdots \mathrm{O} 1^{\mathrm{iii}}$ & 0.95 & 2.43 & $3.104(2)$ & 128 \\
$\mathrm{C} 20-\mathrm{H} 20 \cdots 3^{\text {iv }}$ & 0.95 & 2.33 & $3.176(2)$ & 147 \\
$\mathrm{C} 8-\mathrm{H} 8 B \cdots C g 6^{\mathrm{v}}$ & 0.98 & 2.77 & $3.634(2)$ & 147 \\
$\mathrm{C} 24-\mathrm{H} 24 A \cdots \mathrm{Cg}^{\mathrm{vi}}$ & 0.98 & 2.77 & $3.628(2)$ & 145 \\
\hline
\end{tabular}

Symmetry codes: (i) $-x+2,-y,-z$; (ii) $x+1, y, z-1$; (iii) $-x-1,-y+1,-z+1$; (iv) $-x+1,-y,-z+1$; (v) $-x,-y+1,-z+1$; (vi) $x+1, y, z$.

loop. Finally, C14-H14 (part of the thiophene ring) forms a bond to $\mathrm{O} 4$ in another nearby $\mathrm{C} 15$-molecule. The $\mathrm{C} 15 \mathrm{~mol}-$ ecules in turn link to further pairs of $\mathrm{C} 1$-molecules and hence form [101] chains. The second chain in (III) (Fig. 10) features the donor groups of the C15-molecule; the $\mathrm{C} 17-\mathrm{H} 17$ (to O1) and $\mathrm{C} 20-\mathrm{H} 20$ (to O3) bonds arise from different sides of the benzene ring and both the acceptor atoms are parts of $\mathrm{C} 1$ molecules: the end result is a [2 $\overline{1} 0]$ chain of alternating $\mathrm{C} 1$ - and C15-molecules. Taken together, a complex three-dimensional network arises, which may be consolidated by a pair of weak $\mathrm{C}-\mathrm{H} \cdots \pi$ interactions arising from methyl groups, assuming that the $\mathrm{H}$ atoms in question have been reliably located.

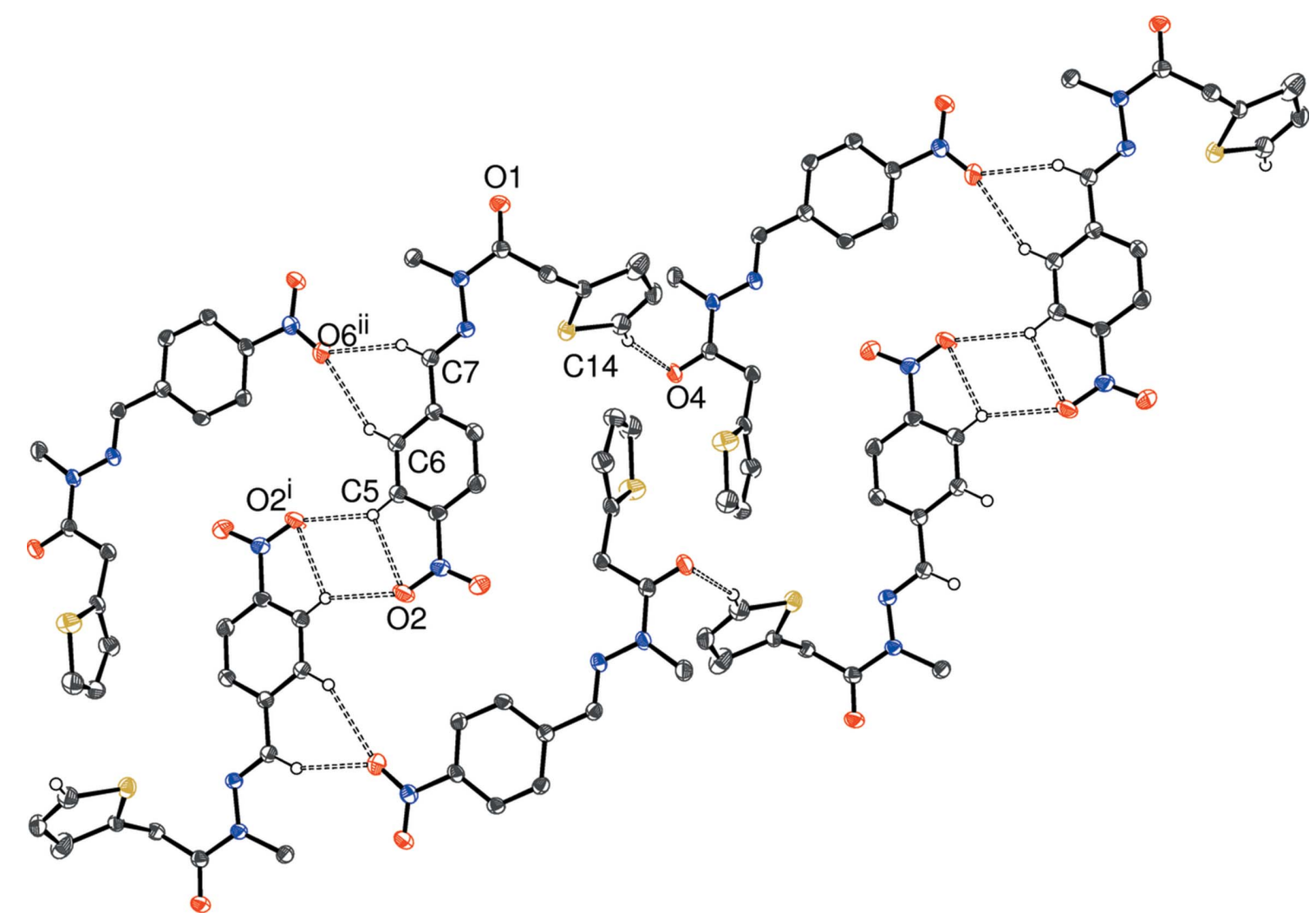

Figure 8

Fragment of a [101] hydrogen-bonded chain in the crystal of (III). [Symmetry codes: (i) $2-x,-y,-z$; (ii) $x+1, y, z-1$.] All $\mathrm{H}$ atoms not involved in hydrogen bonds have been omitted for clarity. 


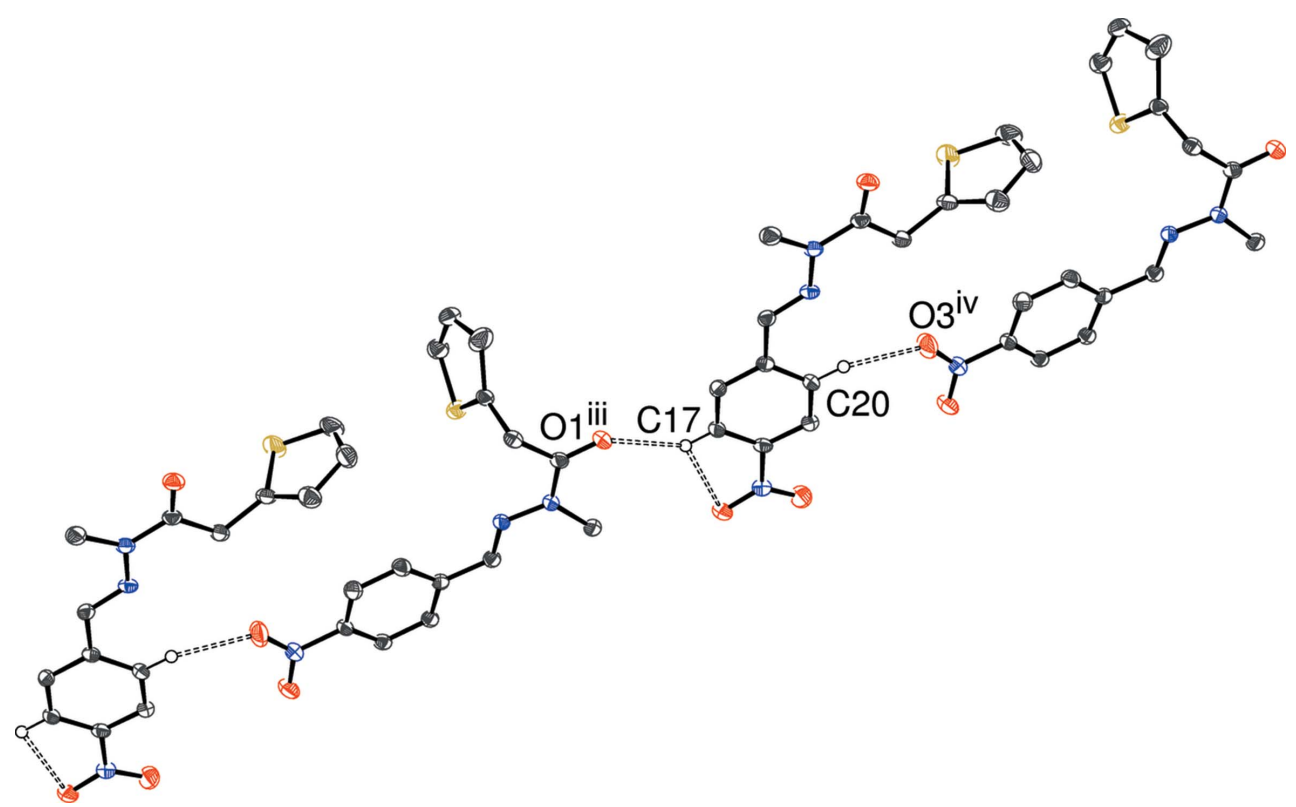

Figure 9

Fragment of a [210] hydrogen-bonded chain in the crystal of (III). [Symmetry codes: (iii) $1-x, 1-y, 1-z ;$ (iv) $1-x,-y, 1-z$.] All H atoms not involved in hydrogen bonds have been omitted for clarity.

\section{Database survey}

A survey of the Cambridge Structural Database (V5.37, last update May 2016; Groom et al., 2016) for the common central $-\mathrm{CH}=\mathrm{N}-\mathrm{N}\left(\mathrm{CH}_{3}\right)-\mathrm{C}(=\mathrm{O})-\mathrm{CH}_{2}-$ fragment of the title compounds revealed just three matches, viz. FOTMUX (Ramirez et al., 2009a), KULREP (Ramirez et al., 2009b) and OFEBIL (Cao et al., 2007). FOTMUX is an interesting binuclear copper complex but none of these materials have a close relationship to the isomeric compounds reported here.

\section{Synthesis and crystallization}

The appropriate derivative (Cardoso et al., 2014) of (1) (0.2 g, 1.0 equivalent) was suspended in acetone $(5.0 \mathrm{ml})$ and potassium carbonate (4.0 equivalents) was added. The reaction mixture was stirred at room temperature for $30 \mathrm{~min}$ and methyl iodide (4.0 equivalents) was added. The reaction mixture was maintained at $313 \mathrm{~K}$, until thin-layer chromatography indicated that the reaction was complete. The reaction mixture was rotary evaporated to leave a residue, which was dissolved in water $(20.0 \mathrm{ml})$ and extracted with ethyl acetate $(3 \times 10.0 \mathrm{ml})$. The organic phases were combined, dried with anhydrous $\mathrm{MgSO}_{4}$, filtered and then evaporated at reduced pressure. The crystals used for intensity data collection were recrystallized from ethanol solution.

$(E)-N$-Methyl- $N^{\prime}$-(2-nitrophenylmethylidene)-2-(thiophen2-yl)acetohydrazide, (I); yield: 57\%; yellow solid; m.p. 366367 K. ${ }^{1} \mathrm{H}$ NMR (400 MHz, DMSO): $\delta 8.21(1 \mathrm{H} ; s ; \mathrm{N}=\mathrm{CH})$, $8.12\left(1 \mathrm{H} ; d d ; J_{\mathrm{HH}}=8.0\right.$ and $\left.1.2 \mathrm{~Hz} ; \mathrm{H}-11^{\prime}\right), 8.04\left(1 \mathrm{H} ; d d ; J_{\mathrm{HH}}=\right.$ 8.4 and $\left.0.8 \mathrm{~Hz} ; \mathrm{H}-8^{\prime}\right), 7.83-7.80\left(1 \mathrm{H} ; m ; \mathrm{H}-10^{\prime}\right), 7.69-7.67(1 \mathrm{H}$; $m$; H-9') $7.37\left(1 \mathrm{H} ; d d ; J_{\mathrm{HH}}=4.8\right.$ and $\left.1.6 \mathrm{~Hz} ; \mathrm{H}-5\right) 6.96-6.94$ $(2 \mathrm{H} ; m ; \mathrm{H}-3$ and $\mathrm{H}-4), 4.34\left(2 \mathrm{H} ; s ; \mathrm{CH}_{2}\right), 3.32\left(3 \mathrm{H} ; s ; \mathrm{N}-\mathrm{CH}_{3}\right)$. ${ }^{13} \mathrm{C}$ NMR (125 MHz; DMSO): $\delta 171.0(\mathrm{C}=\mathrm{O}), 148.3\left(\mathrm{C}-7^{\prime}\right)$,
$136.8(\mathrm{~N}=\mathrm{CH}), 136.1(\mathrm{C}-2), 133.4\left(\mathrm{C}-10^{\prime}\right), 130.4\left(\mathrm{C}-9^{\prime}\right), 128.8$ (C-11'), 128.3 (C-6'), 126.8 (C-3), 126.5 (C-4), 125.2 (C-5), $124.5\left(\mathrm{C}-8^{\prime}\right), 33.9\left(\mathrm{~N}-\mathrm{CH}_{3}\right), 28.1\left(\mathrm{CH}_{2}\right)$. MS/ESI: $[M+\mathrm{Na}]: 326$. IR $v_{\max }\left(\mathrm{cm}^{-1} ; \mathrm{KBr}\right.$ pellet $): 1681(\mathrm{C}=\mathrm{O}) ; 3088\left(\mathrm{~N}-\mathrm{CH}_{3}\right)$.

(E)-N-Methyl- $N^{\prime}$-(3-nitrophenylmethylidene)-2-(thiophen2-yl)acetohydrazide, (II); yield: 73\%; yellow solid; m.p. 378383 K. 1H NMR (400 MHz, DMSO): $\delta 8.61(1 \mathrm{H} ; s ; \mathrm{N}=\mathrm{CH})$, 8.29-8.25 (2H; $m$; H-11' and H-9'), $8.17\left(1 \mathrm{H} ; s ; \mathrm{H}-7^{\prime}\right), 7.79-7.75$ $\left(1 \mathrm{H} ; m ; \mathrm{H}-10^{\prime}\right), 7.37-7.35(1 \mathrm{H} ; m ; \mathrm{H}-5), 7.00-6.99(1 \mathrm{H} ; m ; \mathrm{H}-4)$ 6.96-6.94 (1H; $m ; \mathrm{H}-3), 4.40\left(2 \mathrm{H} ; s ; \mathrm{CH}_{2}\right), 3.35\left(3 \mathrm{H} ; s ; \mathrm{N}-\mathrm{CH}_{3}\right)$. ${ }^{13} \mathrm{C}$ NMR (125 MHz; DMSO) $\delta: 170.9(\mathrm{C}=\mathrm{O}), 148.2\left(\mathrm{C}-8^{\prime}\right)$, $138.6(\mathrm{~N}=\mathrm{CH}), 136.9(\mathrm{C}-2), 136.5\left(\mathrm{C}-6^{\prime}\right), 132.8\left(\mathrm{C}-11^{\prime}\right), 130.4$ (C-10'), 126.7 (C-9'), 126.6 (C-3), 125.2 (C-4), 123.9 (C-5), $121.6\left({\mathrm{C}-7^{\prime}}^{\prime}\right), 34.3\left(\mathrm{~N}-\mathrm{CH}_{3}\right), 28.2\left(\mathrm{CH}_{2}\right)$. MS/ESI: $[M+\mathrm{Na}]: 326$. IR $v_{\text {max }}\left(\mathrm{cm}^{-1}\right.$; KBr pellet): $1668(\mathrm{C}=\mathrm{O}) ; 2962\left(\mathrm{~N}-\mathrm{CH}_{3}\right)$.

(E)-N-Methyl- $N^{\prime}$-(4-nitrophenylmethylidene)-2-(thiophen2-yl)acetohydrazide, (III); yield: 55\%; yellow solid; m.p. 428$433 \mathrm{~K} .{ }^{1} \mathrm{H}$ NMR (400 MHz; DMSO) $\delta: 8.32\left(2 \mathrm{H}\right.$; d; $J_{\mathrm{HH}}=$ $8.8 \mathrm{~Hz} ; \mathrm{H}-8^{\prime}$ and $\left.\mathrm{H}-10^{\prime}\right), 8.13(1 \mathrm{H} ; s ; \mathrm{N}=\mathrm{CH}), 8.07\left(2 \mathrm{H} ; \mathrm{d} ; J_{\mathrm{HH}}\right.$ $=8.8 \mathrm{~Hz} ; \mathrm{H}^{-} 7^{\prime}$ and $\left.\mathrm{H}-11^{\prime}\right), 7.36\left(1 \mathrm{H} ; d d ; J_{\mathrm{HH}}=4.8\right.$ and $1.2 \mathrm{~Hz}$ H-5), 7.00-6.99 (1H; $m$; H-3), 6.96-6.94 (1H; $m$; H-4), $4.41(2 \mathrm{H}$; $\left.s ; \mathrm{CH}_{2}\right), 3.36\left(3 \mathrm{H} ; s ; \mathrm{N}-\mathrm{CH}_{3}\right) .{ }^{13} \mathrm{C}$ NMR (125 MHz; DMSO) $\delta$ : $171.0(\mathrm{C}=\mathrm{O}), 147.6\left(\mathrm{C}-9^{\prime}\right), 140.9(\mathrm{~N}=\mathrm{CH}), 138.4\left(\mathrm{C}-6^{\prime}\right), 136.8$ (C-2), 128.0 (C-3), 126.8 (C-4), 126.5 (C-5), 125.2 (C-7' and C$\left.11^{\prime}\right), 124.0\left(\mathrm{C}-\mathrm{C}-8^{\prime}\right.$ and $\left.\mathrm{C}-10^{\prime}\right), 34.2\left(\mathrm{~N}^{-} \mathrm{CH}_{3}\right), 28.3\left(\mathrm{CH}_{2}\right) . \mathrm{MS} /$ ESI: $[M+\mathrm{Na}]: 326$. IR $v_{\max }\left(\mathrm{cm}^{-1} ; \mathrm{KBr}\right.$ pellet $): 1678(\mathrm{C}=\mathrm{O})$; $3101\left(\mathrm{~N}-\mathrm{CH}_{3}\right)$.

\section{Refinement}

Crystal data, data collection and structure refinement details are summarized in Table 4. The $\mathrm{H}$ atoms were placed geometrically $(\mathrm{C}-\mathrm{H}=0.95-1.00 \AA)$ and refined as riding 
Table 4

Experimental details.

(I) (II)

Crystal data

Chemical formula

$M_{\mathrm{r}}$

Crystal system, space group

Temperature (K)

$a, b, c(\AA)$

$\alpha, \beta, \gamma\left({ }^{\circ}\right)$

$V\left(\AA^{3}\right)$

Z

Radiation type

$\mu\left(\mathrm{mm}^{-1}\right)$

Crystal size (mm)

Data collection

Diffractometer

No. of measured, independent and

observed $[I>2 \sigma(I)]$ reflections

$R_{\text {int }}$

$(\sin \theta / \lambda)_{\max }\left(\AA^{-1}\right)$

\section{$\mathrm{C}_{14} \mathrm{H}_{13} \mathrm{~N}_{3} \mathrm{O}_{3} \mathrm{~S}$}

303.33

Monoclinic, $P 2_{1} / c$

100

7.3989 (5), 24.4910 (17), 7.7126 (5)

90, $96.022(2), 90$

$1389.86(16)$

4

Mo $K \alpha$

0.25

$0.08 \times 0.07 \times 0.03$

Rigaku Mercury CCD

9379, 3157, 2439

0.040

0.648

$0.041,0.106,1.05$

3157

192

$\mathrm{H}$-atom parameters constrained $0.45,-0.35$
(III)

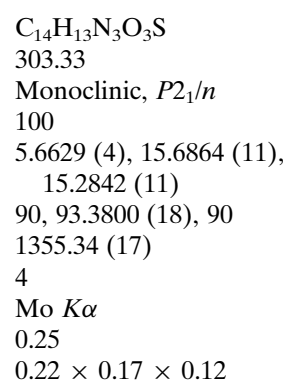

$\mathrm{C}_{14} \mathrm{H}_{13} \mathrm{~N}_{3} \mathrm{O}_{3} \mathrm{~S}$

303.33

Triclinic, $P \overline{1}$

100

6.1893 (4), 12.9177 (9),

$17.3828(12)$

93.995 (7), 90.386 (6), 95.963 (7)

$1378.77(16)$

Mo $K \alpha$

0.25

$0.20 \times 0.18 \times 0.16$

Rigaku Mercury CCD

9365, 3110, 2757

0.031

0.649

Rigaku Mercury CCD
$18534,6279,4868$

0.078
0.649

$0.058,0.166,1.10$
6279
383
H-atom parameters constrained
$0.67,-0.61$

\begin{tabular}{ll}
$0.034,0.096,1.08$ & $0.058,0.166,1.10$ \\
3110 & 6279 \\
191 & 383 \\
H-atom parameters constrained & H-atom parameters constrained \\
$0.30,-0.28$ & $0.67,-0.61$ \\
\hline
\end{tabular}

Computer programs: CrystalClear (Rigaku, 2012), SHELXS97 (Sheldrick, 2008), SHELXL2014 (Sheldrick, 2015), ORTEP-3 for Windows (Farrugia, 2012) and publCIF (Westrip, 2010).

atoms. The constraint $U_{\text {iso }}(\mathrm{H})=1.2 U_{\text {eq }}$ (carrier) or $1.5 U_{\text {eq }}$ (methyl) was applied in all cases. The methyl group was allowed to rotate, but not to tip, to best fit the electron density (AFIX 137 instruction). In each case, this group rotated from its initial orientation to minimize steric interaction with atom $\mathrm{H} 7$; the final orientation leads to a short $\mathrm{C} 8-\mathrm{H} \cdots \mathrm{O} 1$ intramolecular contact but we do not regard this as a bond. The thiophene rings in (I) and (III) show 'flip' rotational disorder.

\section{Acknowledgements}

We thank the EPSRC National Crystallography Service (University of Southampton) for X-ray data collection.

\section{References}

Cao, X.-Y., Harrowfield, J., Nitschke, J., Ramirez, J., Stadler, A.-M., Kyritsakas-Gruber, N., Madalan, A., Rissanen, K., Russo, L., Vaughan, G. \& Lehn, J.-M. (2007). Eur. J. Inorg. Chem. pp. 29442965.
Cardoso, L. N. F., Bispo, M. L. F., Kaiser, C. R., Wardell, J. L., Wardell, S. M. S. V., Lourenço, M. C. S. S., Bezerra, F. A. F., Soares, R. P. P., Rocha, M. N. \& de Souza, M. V. N. (2014). Arch. Pharm. Chem. Life Sci. 347, 432-448.

Farrugia, L. J. (2012). J. Appl. Cryst. 45, 849-854.

Groom, C. R., Bruno, I. J., Lightfoot, M. P. \& Ward, S. C. (2016). Acta Cryst. B72, 171-179.

Ramirez, J., Brelot, L., Osinska, I. \& Stadler, A.-M. (2009b). J. Mol. Struct. 931, 20-24.

Ramirez, J., Stadler, A.-M., Rogez, G., Drillon, M. \& Lehn, J.-M. (2009a). Inorg. Chem. 48, 2456-2463.

Rigaku (2012). CrystalClear. Rigaku Corporation, Tokyo, Japan.

Sheldrick, G. M. (2008). Acta Cryst. A64, 112-122.

Sheldrick, G. M. (2015). Acta Cryst. C71, 3-8.

Sonar, V. N., Parkin, S. \& Crooks, P. A. (2005). Acta Cryst. E61, o933o935.

Souza, M. V. N. de, Ferreira, M. L., Nogueira, T. C. M., Golçalves, R. S. B., Peralta, M. A., Lourenço, M. S. C. F. R. \& Vicente, F. R. (2008). Lett. Drug Des. Discov. 5, 221-224.

Wagner, P., Officer, D. L. \& Kubicki, M. (2006). Acta Cryst. E62, o5931-05932.

Westrip, S. P. (2010). J. Appl. Cryst. 43, 920-925. 


\section{supporting information}

Acta Cryst. (2016). E72, 1677-1682［https://doi.org/10.1107/S2056989016016856]

Different weak interactions in the crystals of three isomeric $(E)-N$-methyl$N^{\prime}$-(nitrobenzylidene)-2-(thiophen-2-yl)acetohydrazides

Laura N. F. Cardoso, Thais C. M. Noguiera, Carlos R. Kaiser, James L. Wardell, Marcus V. N. de Souza, Shaun T. Lancaster and William T. A. Harrison

Computing details

For all compounds, data collection: CrystalClear (Rigaku, 2012); cell refinement: CrystalClear (Rigaku, 2012); data reduction: CrystalClear (Rigaku, 2012); program(s) used to solve structure: SHELXS97 (Sheldrick, 2008); program(s) used to refine structure: SHELXL2014 (Sheldrick, 2015); molecular graphics: ORTEP-3 for Windows (Farrugia, 2012); software used to prepare material for publication: publCIF (Westrip, 2010).

(I) (E)-N-Methyl-N'-(2-nitrobenzylidene)-2-(thiophen-2-yl)acetohydrazide

Crystal data

$\mathrm{C}_{14} \mathrm{H}_{13} \mathrm{~N}_{3} \mathrm{O}_{3} \mathrm{~S}$

$M_{r}=303.33$

Monoclinic, $P 2_{1} / c$

$a=7.3989(5) \AA$

$b=24.4910(17) \AA$

$c=7.7126(5) \AA$

$\beta=96.022(2)^{\circ}$

$V=1389.86(16) \AA^{3}$

$Z=4$

Data collection

Rigaku Mercury CCD

diffractometer

$\omega$ scans

9379 measured reflections

3157 independent reflections

2439 reflections with $I>2 \sigma(I)$

Refinement

Refinement on $F^{2}$

Least-squares matrix: full

$R\left[F^{2}>2 \sigma\left(F^{2}\right)\right]=0.041$

$w R\left(F^{2}\right)=0.106$

$S=1.05$

3157 reflections

192 parameters

0 restraints
$F(000)=632$

$D_{\mathrm{x}}=1.450 \mathrm{Mg} \mathrm{m}^{-3}$

Mo $K \alpha$ radiation, $\lambda=0.71073 \AA$

Cell parameters from 9051 reflections

$\theta=2.5-27.5^{\circ}$

$\mu=0.25 \mathrm{~mm}^{-1}$

$T=100 \mathrm{~K}$

Block, pale yellow

$0.08 \times 0.07 \times 0.03 \mathrm{~mm}$

$R_{\text {int }}=0.040$

$\theta_{\text {max }}=27.4^{\circ}, \theta_{\min }=2.8^{\circ}$

$h=-9 \rightarrow 9$

$k=-31 \rightarrow 28$

$l=-9 \rightarrow 9$

Primary atom site location: structure-invariant direct methods

Hydrogen site location: inferred from neighbouring sites

$\mathrm{H}$-atom parameters constrained

$w=1 /\left[\sigma^{2}\left(F_{0}^{2}\right)+(0.0494 P)^{2}+0.321 P\right]$

where $P=\left(F_{\mathrm{o}}^{2}+2 F_{\mathrm{c}}^{2}\right) / 3$

$(\Delta / \sigma)_{\max }=0.001$

$\Delta \rho_{\max }=0.45$ e $\AA^{-3}$

$\Delta \rho_{\min }=-0.35$ e $\AA^{-3}$ 


\section{Special details}

Geometry. All esds (except the esd in the dihedral angle between two 1.s. planes) are estimated using the full covariance matrix. The cell esds are taken into account individually in the estimation of esds in distances, angles and torsion angles; correlations between esds in cell parameters are only used when they are defined by crystal symmetry. An approximate (isotropic) treatment of cell esds is used for estimating esds involving l.s. planes.

Fractional atomic coordinates and isotropic or equivalent isotropic displacement parameters $\left(\AA^{2}\right)$

\begin{tabular}{|c|c|c|c|c|c|}
\hline & $x$ & $y$ & $z$ & $U_{\text {iso }} * / U_{\text {eq }}$ & Occ. $(<1)$ \\
\hline $\mathrm{C} 1$ & $0.2369(2)$ & $0.49283(6)$ & 0.4509 (2) & 0.0194 (3) & \\
\hline $\mathrm{C} 2$ & $0.2812(2)$ & $0.50348(7)$ & $0.6291(2)$ & 0.0217 (3) & \\
\hline $\mathrm{H} 2$ & 0.2668 & 0.4754 & 0.7116 & $0.026^{*}$ & \\
\hline $\mathrm{C} 3$ & $0.3455(2)$ & $0.55416(7)$ & $0.6873(2)$ & $0.0242(4)$ & \\
\hline H3 & 0.3774 & 0.5601 & 0.8084 & $0.029 *$ & \\
\hline $\mathrm{C} 4$ & 0.3637 (2) & $0.59635(7)$ & $0.5702(2)$ & $0.0244(4)$ & \\
\hline H4 & 0.4078 & 0.6310 & 0.6111 & $0.029 *$ & \\
\hline $\mathrm{C} 5$ & $0.3173(2)$ & $0.58784(7)$ & 0.3934 (2) & $0.0238(4)$ & \\
\hline H5 & 0.3267 & 0.6166 & 0.3122 & $0.029 *$ & \\
\hline C6 & $0.2570(2)$ & $0.53656(7)$ & $0.3373(2)$ & $0.0208(3)$ & \\
\hline C7 & $0.1640(2)$ & $0.43927(6)$ & $0.3917(2)$ & $0.0203(3)$ & \\
\hline H7 & 0.1346 & 0.4320 & 0.2710 & $0.024 *$ & \\
\hline $\mathrm{C} 8$ & $0.0311(2)$ & $0.34081(7)$ & $0.2640(2)$ & $0.0244(4)$ & \\
\hline H8A & 0.1427 & 0.3440 & 0.2066 & $0.037 *$ & \\
\hline H8B & -0.0598 & 0.3665 & 0.2108 & $0.037 *$ & \\
\hline $\mathrm{H} 8 \mathrm{C}$ & -0.0161 & 0.3035 & 0.2504 & $0.037 *$ & \\
\hline C9 & $0.0357(2)$ & $0.31573(7)$ & $0.5737(2)$ & $0.0205(3)$ & \\
\hline $\mathrm{C} 10$ & $0.0923(2)$ & $0.33017(7)$ & $0.7629(2)$ & $0.0218(3)$ & \\
\hline H10A & 0.0681 & 0.3693 & 0.7822 & $0.026^{*}$ & \\
\hline H10B & 0.0197 & 0.3086 & 0.8391 & $0.026^{*}$ & \\
\hline $\mathrm{C} 11$ & $0.2913(2)$ & $0.31855(7)$ & 0.8097 (2) & $0.0235(4)$ & \\
\hline $\mathrm{C} 12$ & $0.39380(13)$ & $0.27005(4)$ & $0.74418(13)$ & $0.0357(3)$ & $0.671(2)$ \\
\hline H12 & 0.3487 & 0.2421 & 0.6658 & $0.043^{*}$ & $0.671(2)$ \\
\hline S1A & $0.39380(13)$ & $0.27005(4)$ & $0.74418(13)$ & $0.0357(3)$ & $0.329(2)$ \\
\hline $\mathrm{C} 13$ & $0.5816(3)$ & $0.27681(9)$ & $0.8322(3)$ & $0.0432(5)$ & \\
\hline H13 & 0.6795 & 0.2541 & 0.8069 & $0.052 *$ & \\
\hline C14 & $0.6023(3)$ & $0.31711(10)$ & $0.9492(3)$ & $0.0499(7)$ & \\
\hline H14 & 0.7135 & 0.3238 & 1.0191 & $0.060 *$ & \\
\hline N1 & $0.14114(17)$ & $0.40276(5)$ & $0.50578(17)$ & $0.0191(3)$ & \\
\hline N2 & $0.07069(18)$ & $0.35331(5)$ & 0.44877 (16) & $0.0194(3)$ & \\
\hline N3 & $0.21802(19)$ & $0.52965(6)$ & $0.14698(17)$ & 0.0239 (3) & \\
\hline $\mathrm{O} 1$ & $-0.03469(16)$ & $0.27167(5)$ & $0.53215(15)$ & $0.0262(3)$ & \\
\hline $\mathrm{O} 2$ & $0.27226(18)$ & $0.48824(5)$ & $0.07864(15)$ & $0.0309(3)$ & \\
\hline $\mathrm{O} 3$ & $0.13598(19)$ & $0.56675(5)$ & $0.06483(16)$ & $0.0341(3)$ & \\
\hline $\mathrm{S} 1$ & $0.42291(8)$ & $0.35327(2)$ & $0.96161(7)$ & $0.0275(2)$ & $0.671(2)$ \\
\hline $\mathrm{C} 12 \mathrm{~A}$ & $0.42291(8)$ & $0.35327(2)$ & $0.96161(7)$ & $0.0275(2)$ & $0.329(2)$ \\
\hline H12A & 0.3995 & 0.3839 & 1.0314 & $0.033^{*}$ & $0.329(2)$ \\
\hline
\end{tabular}


Atomic displacement parameters $\left(\AA^{2}\right)$

\begin{tabular}{lllllll}
\hline & $U^{11}$ & $U^{22}$ & $U^{33}$ & $U^{12}$ & $U^{13}$ & $U^{23}$ \\
\hline C1 & $0.0174(7)$ & $0.0205(8)$ & $0.0205(7)$ & $0.0011(6)$ & $0.0027(6)$ & $0.0006(6)$ \\
C2 & $0.0215(8)$ & $0.0229(8)$ & $0.0207(7)$ & $-0.0004(6)$ & $0.0023(6)$ & $0.0018(6)$ \\
C3 & $0.0229(8)$ & $0.0279(9)$ & $0.0217(8)$ & $-0.0008(7)$ & $0.0021(6)$ & $-0.0041(7)$ \\
C4 & $0.0238(8)$ & $0.0198(8)$ & $0.0301(9)$ & $-0.0013(7)$ & $0.0047(7)$ & $-0.0038(7)$ \\
C5 & $0.0243(8)$ & $0.0202(8)$ & $0.0276(8)$ & $0.0011(7)$ & $0.0070(7)$ & $0.0026(7)$ \\
C6 & $0.0203(8)$ & $0.0228(8)$ & $0.0197(7)$ & $0.0021(6)$ & $0.0034(6)$ & $0.0004(6)$ \\
C7 & $0.0218(8)$ & $0.0208(8)$ & $0.0182(7)$ & $0.0009(6)$ & $0.0014(6)$ & $-0.0006(6)$ \\
C8 & $0.0296(9)$ & $0.0220(8)$ & $0.0207(8)$ & $-0.0020(7)$ & $-0.0018(7)$ & $-0.0023(6)$ \\
C9 & $0.0162(7)$ & $0.0198(8)$ & $0.0251(8)$ & $0.0014(6)$ & $0.0005(6)$ & $0.0014(6)$ \\
C10 & $0.0233(8)$ & $0.0217(8)$ & $0.0205(7)$ & $0.0009(7)$ & $0.0026(6)$ & $0.0032(6)$ \\
C11 & $0.0251(9)$ & $0.0235(9)$ & $0.0210(7)$ & $-0.0045(7)$ & $-0.0016(6)$ & $0.0060(6)$ \\
C12 & $0.0237(5)$ & $0.0446(6)$ & $0.0375(6)$ & $0.0074(4)$ & $-0.0027(4)$ & $-0.0100(4)$ \\
S1A & $0.0237(5)$ & $0.0446(6)$ & $0.0375(6)$ & $0.0074(4)$ & $-0.0027(4)$ & $-0.0100(4)$ \\
C13 & $0.0219(9)$ & $0.0369(11)$ & $0.0710(15)$ & $0.0058(8)$ & $0.0058(9)$ & $0.0196(11)$ \\
C14 & $0.0382(12)$ & $0.0756(17)$ & $0.0322(10)$ & $-0.0314(11)$ & $-0.0139(9)$ & $0.0259(11)$ \\
N1 & $0.0174(6)$ & $0.0181(7)$ & $0.0214(6)$ & $0.0002(5)$ & $0.0005(5)$ & $-0.0007(5)$ \\
N2 & $0.0210(7)$ & $0.0176(7)$ & $0.0188(6)$ & $-0.0009(5)$ & $-0.0020(5)$ & $-0.0007(5)$ \\
N3 & $0.0268(7)$ & $0.0231(7)$ & $0.0227(7)$ & $0.0008(6)$ & $0.0060(6)$ & $0.0027(6)$ \\
O1 & $0.0269(6)$ & $0.0213(6)$ & $0.0295(6)$ & $-0.0040(5)$ & $-0.0008(5)$ & $0.0013(5)$ \\
O2 & $0.0457(8)$ & $0.0252(6)$ & $0.0228(6)$ & $0.0053(6)$ & $0.0083(5)$ & $-0.0007(5)$ \\
O3 & $0.0435(8)$ & $0.0325(7)$ & $0.0259(6)$ & $0.0114(6)$ & $0.0014(6)$ & $0.0069(5)$ \\
S1 & $0.0298(3)$ & $0.0282(3)$ & $0.0251(3)$ & $-0.0060(2)$ & $0.0056(2)$ & $-0.0042(2)$ \\
C12A & $0.0298(3)$ & $0.0282(3)$ & $0.0251(3)$ & $-0.0060(2)$ & $0.0056(2)$ & $-0.0042(2)$ \\
& & & & & & \\
& & & & &
\end{tabular}

Geometric parameters $\left(\AA,{ }^{\circ}\right)$

\begin{tabular}{llll}
\hline $\mathrm{C} 1-\mathrm{C} 6$ & $1.402(2)$ & $\mathrm{C} 9-\mathrm{C} 10$ & $1.517(2)$ \\
$\mathrm{C} 1-\mathrm{C} 2$ & $1.404(2)$ & $\mathrm{C} 10-\mathrm{C} 11$ & $1.506(2)$ \\
$\mathrm{C} 1-\mathrm{C} 7$ & $1.473(2)$ & $\mathrm{C} 10-\mathrm{H} 10 \mathrm{~A}$ & 0.9900 \\
$\mathrm{C} 2-\mathrm{C} 3$ & $1.387(2)$ & $\mathrm{C} 10-\mathrm{H} 10 \mathrm{~B}$ & 0.9900 \\
$\mathrm{C} 2-\mathrm{H} 2$ & 0.9500 & $\mathrm{C} 11-\mathrm{S} 1 \mathrm{~A}$ & $1.524(2)$ \\
$\mathrm{C} 3-\mathrm{C} 4$ & $1.388(2)$ & $\mathrm{C} 11-\mathrm{C} 12$ & $1.524(2)$ \\
$\mathrm{C} 3-\mathrm{H} 3$ & 0.9500 & $\mathrm{C} 11-\mathrm{C} 12 \mathrm{~A}$ & $1.6748(17)$ \\
$\mathrm{C} 4-\mathrm{C} 5$ & $1.386(2)$ & $\mathrm{C} 11-\mathrm{S} 1$ & $1.6748(17)$ \\
$\mathrm{C} 4-\mathrm{H} 4$ & 0.9500 & $\mathrm{C} 12-\mathrm{C} 13$ & $1.490(2)$ \\
$\mathrm{C} 5-\mathrm{C} 6$ & $1.387(2)$ & $\mathrm{C} 12-\mathrm{H} 12$ & 0.9500 \\
$\mathrm{C} 5-\mathrm{H} 5$ & 0.9500 & $\mathrm{~S} 1 \mathrm{~A}-\mathrm{C} 13$ & $1.490(2)$ \\
$\mathrm{C} 6-\mathrm{N} 3$ & $1.476(2)$ & $\mathrm{C} 13-\mathrm{C} 14$ & $1.335(3)$ \\
$\mathrm{C} 7-\mathrm{N} 1$ & $1.278(2)$ & $\mathrm{C} 13-\mathrm{H} 13$ & 0.9500 \\
$\mathrm{C} 7-\mathrm{H} 7$ & 0.9500 & $\mathrm{C} 14-\mathrm{C} 12 \mathrm{~A}$ & $1.607(3)$ \\
$\mathrm{C} 8-\mathrm{N} 2$ & $1.4568(19)$ & $\mathrm{C} 14-\mathrm{S} 1$ & $1.607(3)$ \\
$\mathrm{C} 8-\mathrm{H} 8 \mathrm{~A}$ & 0.9800 & $\mathrm{C} 14-\mathrm{H} 14$ & 0.9500 \\
$\mathrm{C} 8-\mathrm{H} 8 \mathrm{~B}$ & 0.9800 & $\mathrm{~N} 1-\mathrm{N} 2$ & $1.3725(18)$ \\
$\mathrm{C} 8-\mathrm{H} 8 \mathrm{C}$ & 0.9800 & $\mathrm{~N} 3-\mathrm{O} 2$ & $1.2294(18)$ \\
$\mathrm{C} 9-\mathrm{O} 1$ & $1.2262(19)$ & $\mathrm{N} 3-\mathrm{O} 3$ & $1.2308(18)$
\end{tabular}




\begin{tabular}{|c|c|c|c|}
\hline $\mathrm{C} 9-\mathrm{N} 2$ & $1.377(2)$ & $\mathrm{C} 12 \mathrm{~A}-\mathrm{H} 12 \mathrm{~A}$ & 0.9500 \\
\hline $\mathrm{C} 6-\mathrm{C} 1-\mathrm{C} 2$ & $116.18(15)$ & $\mathrm{C} 11-\mathrm{C} 10-\mathrm{H} 10 \mathrm{~B}$ & 109.5 \\
\hline $\mathrm{C} 6-\mathrm{C} 1-\mathrm{C} 7$ & $123.10(14)$ & $\mathrm{C} 9-\mathrm{C} 10-\mathrm{H} 10 \mathrm{~B}$ & 109.5 \\
\hline $\mathrm{C} 2-\mathrm{C} 1-\mathrm{C} 7$ & $120.63(14)$ & $\mathrm{H} 10 \mathrm{~A}-\mathrm{C} 10-\mathrm{H} 10 \mathrm{~B}$ & 108.1 \\
\hline $\mathrm{C} 3-\mathrm{C} 2-\mathrm{C} 1$ & $121.32(15)$ & $\mathrm{C} 10-\mathrm{C} 11-\mathrm{S} 1 \mathrm{~A}$ & $125.17(13)$ \\
\hline $\mathrm{C} 3-\mathrm{C} 2-\mathrm{H} 2$ & 119.3 & $\mathrm{C} 10-\mathrm{C} 11-\mathrm{C} 12$ & $125.17(13)$ \\
\hline $\mathrm{C} 1-\mathrm{C} 2-\mathrm{H} 2$ & 119.3 & $\mathrm{C} 10-\mathrm{C} 11-\mathrm{C} 12 \mathrm{~A}$ & $123.64(13)$ \\
\hline $\mathrm{C} 2-\mathrm{C} 3-\mathrm{C} 4$ & $120.62(15)$ & $\mathrm{S} 1 \mathrm{~A}-\mathrm{C} 11-\mathrm{C} 12 \mathrm{~A}$ & $110.77(11)$ \\
\hline $\mathrm{C} 2-\mathrm{C} 3-\mathrm{H} 3$ & 119.7 & $\mathrm{C} 10-\mathrm{C} 11-\mathrm{S} 1$ & $123.64(13)$ \\
\hline $\mathrm{C} 4-\mathrm{C} 3-\mathrm{H} 3$ & 119.7 & $\mathrm{C} 12-\mathrm{C} 11-\mathrm{S} 1$ & $110.77(11)$ \\
\hline $\mathrm{C} 5-\mathrm{C} 4-\mathrm{C} 3$ & $119.79(15)$ & $\mathrm{C} 13-\mathrm{C} 12-\mathrm{C} 11$ & $103.59(13)$ \\
\hline $\mathrm{C} 5-\mathrm{C} 4-\mathrm{H} 4$ & 120.1 & $\mathrm{C} 13-\mathrm{C} 12-\mathrm{H} 12$ & 128.2 \\
\hline $\mathrm{C} 3-\mathrm{C} 4-\mathrm{H} 4$ & 120.1 & $\mathrm{C} 11-\mathrm{C} 12-\mathrm{H} 12$ & 128.2 \\
\hline $\mathrm{C} 4-\mathrm{C} 5-\mathrm{C} 6$ & $118.79(15)$ & $\mathrm{C} 13-\mathrm{S} 1 \mathrm{~A}-\mathrm{C} 11$ & $103.59(13)$ \\
\hline $\mathrm{C} 4-\mathrm{C} 5-\mathrm{H} 5$ & 120.6 & $\mathrm{C} 14-\mathrm{C} 13-\mathrm{C} 12$ & $115.17(18)$ \\
\hline $\mathrm{C} 6-\mathrm{C} 5-\mathrm{H} 5$ & 120.6 & $\mathrm{C} 14-\mathrm{C} 13-\mathrm{S} 1 \mathrm{~A}$ & $115.17(18)$ \\
\hline $\mathrm{C} 5-\mathrm{C} 6-\mathrm{C} 1$ & $123.27(15)$ & $\mathrm{C} 14-\mathrm{C} 13-\mathrm{H} 13$ & 122.4 \\
\hline $\mathrm{C} 5-\mathrm{C} 6-\mathrm{N} 3$ & $115.94(14)$ & $\mathrm{C} 12-\mathrm{C} 13-\mathrm{H} 13$ & 122.4 \\
\hline $\mathrm{C} 1-\mathrm{C} 6-\mathrm{N} 3$ & $120.78(14)$ & $\mathrm{C} 13-\mathrm{C} 14-\mathrm{C} 12 \mathrm{~A}$ & $114.26(16)$ \\
\hline $\mathrm{N} 1-\mathrm{C} 7-\mathrm{C} 1$ & $118.69(14)$ & $\mathrm{C} 13-\mathrm{C} 14-\mathrm{S} 1$ & $114.26(16)$ \\
\hline $\mathrm{N} 1-\mathrm{C} 7-\mathrm{H} 7$ & 120.7 & $\mathrm{C} 13-\mathrm{C} 14-\mathrm{H} 14$ & 122.9 \\
\hline $\mathrm{C} 1-\mathrm{C} 7-\mathrm{H} 7$ & 120.7 & $\mathrm{~S} 1-\mathrm{C} 14-\mathrm{H} 14$ & 122.9 \\
\hline $\mathrm{N} 2-\mathrm{C} 8-\mathrm{H} 8 \mathrm{~A}$ & 109.5 & $\mathrm{C} 7-\mathrm{N} 1-\mathrm{N} 2$ & $118.03(13)$ \\
\hline $\mathrm{N} 2-\mathrm{C} 8-\mathrm{H} 8 \mathrm{~B}$ & 109.5 & $\mathrm{~N} 1-\mathrm{N} 2-\mathrm{C} 9$ & $117.30(12)$ \\
\hline $\mathrm{H} 8 \mathrm{~A}-\mathrm{C} 8-\mathrm{H} 8 \mathrm{~B}$ & 109.5 & $\mathrm{~N} 1-\mathrm{N} 2-\mathrm{C} 8$ & $121.97(13)$ \\
\hline $\mathrm{N} 2-\mathrm{C} 8-\mathrm{H} 8 \mathrm{C}$ & 109.5 & $\mathrm{C} 9-\mathrm{N} 2-\mathrm{C} 8$ & $120.73(13)$ \\
\hline $\mathrm{H} 8 \mathrm{~A}-\mathrm{C} 8-\mathrm{H} 8 \mathrm{C}$ & 109.5 & $\mathrm{O} 2-\mathrm{N} 3-\mathrm{O} 3$ & $123.62(14)$ \\
\hline $\mathrm{H} 8 \mathrm{~B}-\mathrm{C} 8-\mathrm{H} 8 \mathrm{C}$ & 109.5 & $\mathrm{O} 2-\mathrm{N} 3-\mathrm{C} 6$ & $118.85(13)$ \\
\hline $\mathrm{O} 1-\mathrm{C} 9-\mathrm{N} 2$ & $120.74(14)$ & $\mathrm{O} 3-\mathrm{N} 3-\mathrm{C} 6$ & $117.50(14)$ \\
\hline $\mathrm{O} 1-\mathrm{C} 9-\mathrm{C} 10$ & $121.57(14)$ & $\mathrm{C} 14-\mathrm{S} 1-\mathrm{C} 11$ & $95.85(10)$ \\
\hline $\mathrm{N} 2-\mathrm{C} 9-\mathrm{C} 10$ & $117.66(14)$ & $\mathrm{C} 14-\mathrm{C} 12 \mathrm{~A}-\mathrm{C} 11$ & $95.85(10)$ \\
\hline $\mathrm{C} 11-\mathrm{C} 10-\mathrm{C} 9$ & $110.52(13)$ & $\mathrm{C} 14-\mathrm{C} 12 \mathrm{~A}-\mathrm{H} 12 \mathrm{~A}$ & 132.1 \\
\hline $\mathrm{C} 11-\mathrm{C} 10-\mathrm{H} 10 \mathrm{~A}$ & 109.5 & $\mathrm{C} 11-\mathrm{C} 12 \mathrm{~A}-\mathrm{H} 12 \mathrm{~A}$ & 132.1 \\
\hline $\mathrm{C} 9-\mathrm{C} 10-\mathrm{H} 10 \mathrm{~A}$ & 109.5 & & \\
\hline $\mathrm{C} 6-\mathrm{C} 1-\mathrm{C} 2-\mathrm{C} 3$ & $-1.6(2)$ & $\mathrm{C} 12 \mathrm{~A}-\mathrm{C} 11-\mathrm{S} 1 \mathrm{~A}-\mathrm{C} 13$ & $-5.65(15)$ \\
\hline $\mathrm{C} 7-\mathrm{C} 1-\mathrm{C} 2-\mathrm{C} 3$ & $-178.31(15)$ & $\mathrm{C} 11-\mathrm{C} 12-\mathrm{C} 13-\mathrm{C} 14$ & $6.2(2)$ \\
\hline $\mathrm{C} 1-\mathrm{C} 2-\mathrm{C} 3-\mathrm{C} 4$ & $1.6(3)$ & $\mathrm{C} 11-\mathrm{S} 1 \mathrm{~A}-\mathrm{C} 13-\mathrm{C} 14$ & $6.2(2)$ \\
\hline $\mathrm{C} 2-\mathrm{C} 3-\mathrm{C} 4-\mathrm{C} 5$ & $0.0(3)$ & $\mathrm{S} 1 \mathrm{~A}-\mathrm{C} 13-\mathrm{C} 14-\mathrm{C} 12 \mathrm{~A}$ & $-4.3(2)$ \\
\hline $\mathrm{C} 3-\mathrm{C} 4-\mathrm{C} 5-\mathrm{C} 6$ & $-1.4(2)$ & $\mathrm{C} 12-\mathrm{C} 13-\mathrm{C} 14-\mathrm{S} 1$ & $-4.3(2)$ \\
\hline $\mathrm{C} 4-\mathrm{C} 5-\mathrm{C} 6-\mathrm{C} 1$ & $1.3(2)$ & $\mathrm{C} 1-\mathrm{C} 7-\mathrm{N} 1-\mathrm{N} 2$ & $179.10(13)$ \\
\hline $\mathrm{C} 4-\mathrm{C} 5-\mathrm{C} 6-\mathrm{N} 3$ & $-177.29(14)$ & $\mathrm{C} 7-\mathrm{N} 1-\mathrm{N} 2-\mathrm{C} 9$ & $-175.84(14)$ \\
\hline $\mathrm{C} 2-\mathrm{C} 1-\mathrm{C} 6-\mathrm{C} 5$ & $0.2(2)$ & $\mathrm{C} 7-\mathrm{N} 1-\mathrm{N} 2-\mathrm{C} 8$ & $3.2(2)$ \\
\hline $\mathrm{C} 7-\mathrm{C} 1-\mathrm{C} 6-\mathrm{C} 5$ & $176.78(15)$ & $\mathrm{O} 1-\mathrm{C} 9-\mathrm{N} 2-\mathrm{N} 1$ & $176.75(14)$ \\
\hline $\mathrm{C} 2-\mathrm{C} 1-\mathrm{C} 6-\mathrm{N} 3$ & $178.71(14)$ & $\mathrm{C} 10-\mathrm{C} 9-\mathrm{N} 2-\mathrm{N} 1$ & $-5.0(2)$ \\
\hline $\mathrm{C} 7-\mathrm{C} 1-\mathrm{C} 6-\mathrm{N} 3$ & $-4.7(2)$ & $\mathrm{O} 1-\mathrm{C} 9-\mathrm{N} 2-\mathrm{C} 8$ & $-2.3(2)$ \\
\hline $\mathrm{C} 6-\mathrm{C} 1-\mathrm{C} 7-\mathrm{N} 1$ & $-176.15(15)$ & $\mathrm{C} 10-\mathrm{C} 9-\mathrm{N} 2-\mathrm{C} 8$ & $175.95(14)$ \\
\hline
\end{tabular}




$\begin{array}{llll}\mathrm{C} 2-\mathrm{C} 1-\mathrm{C} 7-\mathrm{N} 1 & 0.3(2) & \mathrm{C} 5-\mathrm{C} 6-\mathrm{N} 3-\mathrm{O} 2 & 135.41(16) \\ \mathrm{O} 1-\mathrm{C} 9-\mathrm{C} 10-\mathrm{C} 11 & 96.54(18) & \mathrm{C} 1-\mathrm{C} 6-\mathrm{N} 3-\mathrm{O} 2 & -43.2(2) \\ \mathrm{N} 2-\mathrm{C} 9-\mathrm{C} 10-\mathrm{C} 11 & -81.73(18) & \mathrm{C} 5-\mathrm{C} 6-\mathrm{N} 3-\mathrm{O} 3 & -42.9(2) \\ \mathrm{C} 9-\mathrm{C} 10-\mathrm{C} 11-\mathrm{S} 1 \mathrm{~A} & -37.7(2) & \mathrm{C} 1-\mathrm{C} 6-\mathrm{N} 3-\mathrm{O} 3 & 138.50(16) \\ \mathrm{C} 9-\mathrm{C} 10-\mathrm{C} 11-\mathrm{C} 12 & -37.7(2) & \mathrm{C} 13-\mathrm{C} 14-\mathrm{S} 1-\mathrm{C} 11 & 0.41(17) \\ \mathrm{C} 9-\mathrm{C} 10-\mathrm{C} 11-\mathrm{C} 12 \mathrm{~A} & 150.50(13) & \mathrm{C} 10-\mathrm{C} 11-\mathrm{S} 1-\mathrm{C} 14 & 176.26(14) \\ \mathrm{C} 9-\mathrm{C} 10-\mathrm{C} 11-\mathrm{S} 1 & 150.50(13) & \mathrm{C} 12-\mathrm{C} 11-\mathrm{S} 1-\mathrm{C} 14 & 3.38(13) \\ \mathrm{C} 10-\mathrm{C} 11-\mathrm{C} 12-\mathrm{C} 13 & -178.39(15) & \mathrm{C} 13-\mathrm{C} 14-\mathrm{C} 12 \mathrm{~A}-\mathrm{C} 11 & 0.41(17) \\ \mathrm{S} 1-\mathrm{C} 11-\mathrm{C} 12-\mathrm{C} 13 & -5.65(15) & \mathrm{C} 10-\mathrm{C} 11-\mathrm{C} 12 \mathrm{~A}-\mathrm{C} 14 & 176.26(14) \\ \mathrm{C} 10-\mathrm{C} 11-\mathrm{S} 1 \mathrm{C}-\mathrm{C} 13 & -178.39(15) & \mathrm{S} 1 \mathrm{~A}-\mathrm{C} 11-\mathrm{C} 12 \mathrm{~A}-\mathrm{C} 14 & 3.38(13)\end{array}$

Hydrogen-bond geometry $\left(A,{ }^{\circ}\right)$

$\mathrm{Cg} 1$ is the centroid of the $\mathrm{S} 1 / \mathrm{C} 11-\mathrm{C} 14$ ring.

\begin{tabular}{lllll}
\hline$D-\mathrm{H}^{2} \cdots A$ & $D-\mathrm{H}$ & $\mathrm{H} \cdots A$ & $D \cdots A$ & $D-\mathrm{H} \cdots A$ \\
\hline $\mathrm{C} 8-\mathrm{H} 8 C \cdots \mathrm{O} 1^{\mathrm{i}}$ & 0.98 & 2.49 & $3.293(2)$ & 139 \\
$\mathrm{C} 10-\mathrm{H} 10 A \cdots \mathrm{O} 3^{\mathrm{ii}}$ & 0.99 & 2.55 & $3.386(2)$ & 142 \\
$\mathrm{C} 10-\mathrm{H} 10 B \cdots 1^{\mathrm{iii}}$ & 0.99 & 2.52 & $3.439(2)$ & 154 \\
$\mathrm{C} 5-\mathrm{H} 5 \cdots C g 1^{\mathrm{iv}}$ & 0.95 & 2.86 & $3.7212(18)$ & 151 \\
$\mathrm{C} 12-\mathrm{H} 12 \cdots C g 1^{\mathrm{i}}$ & 0.95 & 2.85 & $3.5930(13)$ & 136 \\
\hline
\end{tabular}

Symmetry codes: (i) $x,-y+1 / 2, z-1 / 2$; (ii) $-x,-y+1,-z+1$; (iii) $x,-y+1 / 2, z+1 / 2$; (iv) $-x+1,-y+1,-z+1$.

(II) (E)-N-Methyl-N'-(3-nitrobenzylidene)-2-(thiophen-2-yl)acetohydrazide

Crystal data

$\mathrm{C}_{14} \mathrm{H}_{13} \mathrm{~N}_{3} \mathrm{O}_{3} \mathrm{~S}$

$M_{r}=303.33$

Monoclinic, $P 2_{1} / n$

$a=5.6629(4) \AA$

$b=15.6864(11) \AA$

$c=15.2842(11) \AA$

$\beta=93.3800(18)^{\circ}$

$V=1355.34(17) \AA^{3}$

$Z=4$

\section{Data collection}

Rigaku Mercury CCD diffractometer

$\omega$ scans

9365 measured reflections

3110 independent reflections

2757 reflections with $I>2 \sigma(I)$

\section{Refinement}

Refinement on $F^{2}$

Least-squares matrix: full

$R\left[F^{2}>2 \sigma\left(F^{2}\right)\right]=0.034$

$w R\left(F^{2}\right)=0.096$

$S=1.08$

3110 reflections
$F(000)=632$

$D_{\mathrm{x}}=1.487 \mathrm{Mg} \mathrm{m}^{-3}$

Mo $K \alpha$ radiation, $\lambda=0.71073 \AA$

Cell parameters from 9051 reflections

$\theta=2.6-27.5^{\circ}$

$\mu=0.25 \mathrm{~mm}^{-1}$

$T=100 \mathrm{~K}$

Block, yellow

$0.22 \times 0.17 \times 0.12 \mathrm{~mm}$

$R_{\text {int }}=0.031$

$\theta_{\text {max }}=27.5^{\circ}, \theta_{\text {min }}=2.6^{\circ}$

$h=-7 \rightarrow 7$

$k=-20 \rightarrow 19$

$l=-19 \rightarrow 19$

191 parameters

0 restraints

Hydrogen site location: inferred from neighbouring sites

$\mathrm{H}$-atom parameters constrained 
$w=1 /\left[\sigma^{2}\left(F_{\mathrm{o}}^{2}\right)+(0.0505 P)^{2}+0.4026 P\right]$

where $P=\left(F_{\mathrm{o}}^{2}+2 F_{\mathrm{c}}^{2}\right) / 3$

$(\Delta / \sigma)_{\max }<0.001$

$$
\Delta \rho_{\max }=0.30 \mathrm{e} \AA^{-3}
$$

\section{Special details}

Geometry. All esds (except the esd in the dihedral angle between two 1.s. planes) are estimated using the full covariance matrix. The cell esds are taken into account individually in the estimation of esds in distances, angles and torsion angles; correlations between esds in cell parameters are only used when they are defined by crystal symmetry. An approximate (isotropic) treatment of cell esds is used for estimating esds involving l.s. planes.

Fractional atomic coordinates and isotropic or equivalent isotropic displacement parameters $\left(\hat{A}^{2}\right)$

\begin{tabular}{|c|c|c|c|c|}
\hline & $x$ & $y$ & $z$ & $U_{\text {iso }} * / U_{\text {eq }}$ \\
\hline $\mathrm{C} 1$ & 0.3945 (2) & $0.25348(8)$ & $0.28563(8)$ & $0.0187(2)$ \\
\hline $\mathrm{C} 2$ & $0.4786(2)$ & $0.33560(8)$ & $0.27161(8)$ & $0.0188(2)$ \\
\hline $\mathrm{H} 2$ & 0.6256 & 0.3540 & 0.2983 & $0.023 *$ \\
\hline $\mathrm{C} 3$ & $0.3425(2)$ & $0.38950(8)$ & $0.21792(8)$ & $0.0192(3)$ \\
\hline $\mathrm{C} 4$ & $0.1240(2)$ & $0.36637(9)$ & $0.17844(8)$ & $0.0212(3)$ \\
\hline $\mathrm{H} 4$ & 0.0333 & 0.4055 & 0.1430 & $0.025 *$ \\
\hline $\mathrm{C} 5$ & $0.0432(2)$ & $0.28453(9)$ & $0.19246(8)$ & $0.0225(3)$ \\
\hline H5 & -0.1045 & 0.2667 & 0.1659 & $0.027^{*}$ \\
\hline C6 & $0.1768(2)$ & $0.22833(8)$ & $0.24516(8)$ & $0.0210(3)$ \\
\hline H6 & 0.1202 & 0.1721 & 0.2539 & $0.025 *$ \\
\hline C7 & $0.5216(2)$ & $0.19343(8)$ & $0.34489(8)$ & 0.0198 (3) \\
\hline $\mathrm{H} 7$ & 0.4668 & 0.1365 & 0.3494 & $0.024 *$ \\
\hline $\mathrm{C} 8$ & $0.7162(2)$ & $0.07490(8)$ & $0.45928(9)$ & $0.0225(3)$ \\
\hline H8A & 0.5539 & 0.0795 & 0.4777 & $0.034^{*}$ \\
\hline $\mathrm{H} 8 \mathrm{~B}$ & 0.8134 & 0.0441 & 0.5040 & $0.034^{*}$ \\
\hline $\mathrm{H} 8 \mathrm{C}$ & 0.7160 & 0.0439 & 0.4036 & $0.034 *$ \\
\hline C9 & $1.0134(2)$ & $0.18638(9)$ & $0.49611(8)$ & 0.0199 (3) \\
\hline $\mathrm{C} 10$ & $1.1004(2)$ & $0.27656(8)$ & $0.48044(8)$ & $0.0211(3)$ \\
\hline H10A & 1.2731 & 0.2785 & 0.4947 & $0.025^{*}$ \\
\hline H10B & 1.0732 & 0.2901 & 0.4174 & $0.025^{*}$ \\
\hline C11 & $0.9841(2)$ & $0.34407(8)$ & $0.53279(8)$ & 0.0195 (3) \\
\hline $\mathrm{C} 12$ & $1.0854(2)$ & $0.38920(9)$ & $0.60202(9)$ & $0.0239(3)$ \\
\hline H12 & 1.2409 & 0.3788 & 0.6265 & $0.029 *$ \\
\hline $\mathrm{C} 13$ & $0.9330(2)$ & $0.45351(9)$ & $0.63371(9)$ & $0.0248(3)$ \\
\hline H13 & 0.9760 & 0.4907 & 0.6810 & $0.030^{*}$ \\
\hline C14 & $0.7188(2)$ & $0.45511(9)$ & $0.58811(9)$ & $0.0253(3)$ \\
\hline H14 & 0.5950 & 0.4936 & 0.5998 & $0.030 *$ \\
\hline N1 & $0.70671(18)$ & $0.21766(7)$ & $0.39066(7)$ & $0.0189(2)$ \\
\hline N2 & $0.81324(18)$ & $0.15999(7)$ & $0.44806(7)$ & $0.0195(2)$ \\
\hline N3 & 0.43337 (19) & $0.47517(7)$ & $0.20197(7)$ & $0.0213(2)$ \\
\hline $\mathrm{O} 1$ & $1.11711(16)$ & $0.13877(6)$ & $0.54872(6)$ & $0.0251(2)$ \\
\hline $\mathrm{O} 2$ & $0.31572(17)$ & $0.52296(6)$ & $0.15251(6)$ & $0.0262(2)$ \\
\hline $\mathrm{O} 3$ & $0.62386(17)$ & $0.49615(7)$ & $0.23846(7)$ & $0.0291(2)$ \\
\hline S1 & $0.69978(5)$ & $0.37937(2)$ & $0.50723(2)$ & $0.02407(11)$ \\
\hline
\end{tabular}


Atomic displacement parameters $\left(\AA^{2}\right)$

\begin{tabular}{lllllll}
\hline & $U^{11}$ & $U^{22}$ & $U^{33}$ & $U^{12}$ & $U^{13}$ & $U^{23}$ \\
\hline C1 & $0.0173(5)$ & $0.0210(6)$ & $0.0175(5)$ & $0.0019(5)$ & $-0.0020(4)$ & $-0.0014(5)$ \\
C2 & $0.0153(5)$ & $0.0219(6)$ & $0.0186(6)$ & $0.0009(5)$ & $-0.0024(4)$ & $-0.0011(5)$ \\
C3 & $0.0185(6)$ & $0.0195(6)$ & $0.0192(6)$ & $0.0004(5)$ & $-0.0011(5)$ & $-0.0015(5)$ \\
C4 & $0.0183(6)$ & $0.0247(6)$ & $0.0199(6)$ & $0.0036(5)$ & $-0.0040(5)$ & $-0.0003(5)$ \\
C5 & $0.0169(6)$ & $0.0278(7)$ & $0.0221(6)$ & $0.0001(5)$ & $-0.0051(5)$ & $-0.0025(5)$ \\
C6 & $0.0191(6)$ & $0.0218(6)$ & $0.0217(6)$ & $-0.0014(5)$ & $-0.0029(5)$ & $-0.0016(5)$ \\
C7 & $0.0184(5)$ & $0.0194(6)$ & $0.0212(6)$ & $-0.0004(5)$ & $-0.0023(5)$ & $0.0006(5)$ \\
C8 & $0.0229(6)$ & $0.0184(6)$ & $0.0252(6)$ & $-0.0010(5)$ & $-0.0053(5)$ & $0.0022(5)$ \\
C9 & $0.0157(5)$ & $0.0239(6)$ & $0.0199(6)$ & $0.0023(5)$ & $-0.0013(4)$ & $-0.0031(5)$ \\
C10 & $0.0141(5)$ & $0.0250(6)$ & $0.0239(6)$ & $-0.0009(5)$ & $-0.0017(4)$ & $-0.0019(5)$ \\
C11 & $0.0152(5)$ & $0.0207(6)$ & $0.0223(6)$ & $-0.0012(5)$ & $-0.0015(4)$ & $0.0018(5)$ \\
C12 & $0.0182(6)$ & $0.0266(7)$ & $0.0265(7)$ & $-0.0015(5)$ & $-0.0017(5)$ & $-0.0023(5)$ \\
C13 & $0.0217(6)$ & $0.0259(7)$ & $0.0264(6)$ & $-0.0010(5)$ & $-0.0028(5)$ & $-0.0039(5)$ \\
C14 & $0.0232(6)$ & $0.0232(7)$ & $0.0292(7)$ & $0.0029(5)$ & $-0.0010(5)$ & $-0.0023(5)$ \\
N1 & $0.0171(5)$ & $0.0199(5)$ & $0.0193(5)$ & $0.0022(4)$ & $-0.0025(4)$ & $0.0017(4)$ \\
N2 & $0.0179(5)$ & $0.0184(5)$ & $0.0215(5)$ & $0.0005(4)$ & $-0.0055(4)$ & $0.0018(4)$ \\
N3 & $0.0215(5)$ & $0.0212(5)$ & $0.0208(5)$ & $0.0011(4)$ & $-0.0029(4)$ & $-0.0009(4)$ \\
O1 & $0.0215(4)$ & $0.0274(5)$ & $0.0254(5)$ & $0.0037(4)$ & $-0.0077(4)$ & $0.0008(4)$ \\
O2 & $0.0284(5)$ & $0.0215(5)$ & $0.0280(5)$ & $0.0056(4)$ & $-0.0061(4)$ & $0.0028(4)$ \\
O3 & $0.0263(5)$ & $0.0283(5)$ & $0.0312(5)$ & $-0.0069(4)$ & $-0.0104(4)$ & $0.0028(4)$ \\
S1 & $0.01743(17)$ & $0.02608(19)$ & $0.02791(19)$ & $0.00234(12)$ & $-0.00532(13)$ & $-0.00320(13)$ \\
& & & & & & \\
\hline & & & & & &
\end{tabular}

Geometric parameters $\left(\AA,{ }^{\circ}\right)$

\begin{tabular}{llll}
\hline $\mathrm{C} 1-\mathrm{C} 2$ & $1.3943(18)$ & $\mathrm{C} 9-\mathrm{O} 1$ & $1.2220(16)$ \\
$\mathrm{C} 1-\mathrm{C} 6$ & $1.4027(16)$ & $\mathrm{C} 9-\mathrm{N} 2$ & $1.3776(15)$ \\
$\mathrm{C} 1-\mathrm{C} 7$ & $1.4659(17)$ & $\mathrm{C} 9-\mathrm{C} 10$ & $1.5215(18)$ \\
$\mathrm{C} 2-\mathrm{C} 3$ & $1.3811(17)$ & $\mathrm{C} 10-\mathrm{C} 11$ & $1.5025(18)$ \\
$\mathrm{C} 2-\mathrm{H} 2$ & 0.9500 & $\mathrm{C} 10-\mathrm{H} 10 \mathrm{~A}$ & 0.9900 \\
$\mathrm{C} 3-\mathrm{C} 4$ & $1.3923(17)$ & $\mathrm{C} 10-\mathrm{H} 10 \mathrm{~B}$ & 0.9900 \\
$\mathrm{C} 3-\mathrm{N} 3$ & $1.4645(17)$ & $\mathrm{C} 11-\mathrm{C} 12$ & $1.3705(18)$ \\
$\mathrm{C} 4-\mathrm{C} 5$ & $1.3839(19)$ & $\mathrm{C} 11-\mathrm{S} 1$ & $1.7256(12)$ \\
$\mathrm{C} 4-\mathrm{H} 4$ & 0.9500 & $\mathrm{C} 12-\mathrm{C} 13$ & $1.4306(19)$ \\
$\mathrm{C} 5-\mathrm{C} 6$ & $1.3883(18)$ & $\mathrm{C} 12-\mathrm{H} 12$ & 0.9500 \\
$\mathrm{C} 5-\mathrm{H} 5$ & 0.9500 & $\mathrm{C} 13-\mathrm{C} 14$ & $1.3630(18)$ \\
$\mathrm{C} 6-\mathrm{H} 6$ & 0.9500 & $\mathrm{C} 13-\mathrm{H} 13$ & 0.9500 \\
$\mathrm{C} 7-\mathrm{N} 1$ & $1.2829(16)$ & $\mathrm{C} 14-\mathrm{S} 1$ & $1.7134(14)$ \\
$\mathrm{C} 7-\mathrm{H} 7$ & 0.9500 & $\mathrm{C} 14-\mathrm{H} 14$ & 1.9500 \\
$\mathrm{C} 8-\mathrm{N} 2$ & $1.4573(16)$ & $\mathrm{N} 1-\mathrm{N} 2$ & $1.2297(14)$ \\
$\mathrm{C} 8-\mathrm{H} 8 \mathrm{~A}$ & 0.9800 & $\mathrm{~N} 3-\mathrm{O} 3$ & $1.2321(14)$ \\
$\mathrm{C} 8-\mathrm{H} 8 \mathrm{~B}$ & 0.9800 & $\mathrm{~N} 3-\mathrm{O} 2$ & \\
$\mathrm{C} 8-\mathrm{H} 8 \mathrm{C}$ & 0.9800 & & $121.63(11)$ \\
& & & $117.35(11)$ \\
$\mathrm{C} 2-\mathrm{C} 1-\mathrm{C} 6$ & $119.43(11)$ & $\mathrm{O} 1-\mathrm{C} 9-\mathrm{C} 10$ & \\
$\mathrm{C} 2-\mathrm{C} 1-\mathrm{C} 7$ & $121.93(11)$ & $\mathrm{N} 2-\mathrm{C} 9-\mathrm{C} 10$ &
\end{tabular}




\begin{tabular}{|c|c|c|c|}
\hline $\mathrm{C} 6-\mathrm{C} 1-\mathrm{C} 7$ & $118.58(12)$ & $\mathrm{C} 11-\mathrm{C} 10-\mathrm{C} 9$ & $114.54(11)$ \\
\hline $\mathrm{C} 3-\mathrm{C} 2-\mathrm{C} 1$ & $118.24(11)$ & $\mathrm{C} 11-\mathrm{C} 10-\mathrm{H} 10 \mathrm{~A}$ & 108.6 \\
\hline $\mathrm{C} 3-\mathrm{C} 2-\mathrm{H} 2$ & 120.9 & $\mathrm{C} 9-\mathrm{C} 10-\mathrm{H} 10 \mathrm{~A}$ & 108.6 \\
\hline $\mathrm{C} 1-\mathrm{C} 2-\mathrm{H} 2$ & 120.9 & $\mathrm{C} 11-\mathrm{C} 10-\mathrm{H} 10 \mathrm{~B}$ & 108.6 \\
\hline $\mathrm{C} 2-\mathrm{C} 3-\mathrm{C} 4$ & $123.26(12)$ & $\mathrm{C} 9-\mathrm{C} 10-\mathrm{H} 10 \mathrm{~B}$ & 108.6 \\
\hline $\mathrm{C} 2-\mathrm{C} 3-\mathrm{N} 3$ & $118.13(11)$ & $\mathrm{H} 10 \mathrm{~A}-\mathrm{C} 10-\mathrm{H} 10 \mathrm{~B}$ & 107.6 \\
\hline $\mathrm{C} 4-\mathrm{C} 3-\mathrm{N} 3$ & $118.62(11)$ & $\mathrm{C} 12-\mathrm{C} 11-\mathrm{C} 10$ & $126.76(11)$ \\
\hline $\mathrm{C} 5-\mathrm{C} 4-\mathrm{C} 3$ & $117.93(12)$ & $\mathrm{C} 12-\mathrm{C} 11-\mathrm{S} 1$ & $110.59(10)$ \\
\hline $\mathrm{C} 5-\mathrm{C} 4-\mathrm{H} 4$ & 121.0 & $\mathrm{C} 10-\mathrm{C} 11-\mathrm{S} 1$ & $122.55(9)$ \\
\hline $\mathrm{C} 3-\mathrm{C} 4-\mathrm{H} 4$ & 121.0 & $\mathrm{C} 11-\mathrm{C} 12-\mathrm{C} 13$ & $113.09(12)$ \\
\hline $\mathrm{C} 4-\mathrm{C} 5-\mathrm{C} 6$ & $120.32(11)$ & $\mathrm{C} 11-\mathrm{C} 12-\mathrm{H} 12$ & 123.5 \\
\hline $\mathrm{C} 4-\mathrm{C} 5-\mathrm{H} 5$ & 119.8 & $\mathrm{C} 13-\mathrm{C} 12-\mathrm{H} 12$ & 123.5 \\
\hline $\mathrm{C} 6-\mathrm{C} 5-\mathrm{H} 5$ & 119.8 & $\mathrm{C} 14-\mathrm{C} 13-\mathrm{C} 12$ & $112.11(12)$ \\
\hline $\mathrm{C} 5-\mathrm{C} 6-\mathrm{C} 1$ & $120.80(12)$ & $\mathrm{C} 14-\mathrm{C} 13-\mathrm{H} 13$ & 123.9 \\
\hline $\mathrm{C} 5-\mathrm{C} 6-\mathrm{H} 6$ & 119.6 & $\mathrm{C} 12-\mathrm{C} 13-\mathrm{H} 13$ & 123.9 \\
\hline $\mathrm{C} 1-\mathrm{C} 6-\mathrm{H} 6$ & 119.6 & $\mathrm{C} 13-\mathrm{C} 14-\mathrm{S} 1$ & $111.86(11)$ \\
\hline $\mathrm{N} 1-\mathrm{C} 7-\mathrm{C} 1$ & $120.14(12)$ & $\mathrm{C} 13-\mathrm{C} 14-\mathrm{H} 14$ & 124.1 \\
\hline $\mathrm{N} 1-\mathrm{C} 7-\mathrm{H} 7$ & 119.9 & $\mathrm{~S} 1-\mathrm{C} 14-\mathrm{H} 14$ & 124.1 \\
\hline $\mathrm{C} 1-\mathrm{C} 7-\mathrm{H} 7$ & 119.9 & $\mathrm{C} 7-\mathrm{N} 1-\mathrm{N} 2$ & $117.82(11)$ \\
\hline $\mathrm{N} 2-\mathrm{C} 8-\mathrm{H} 8 \mathrm{~A}$ & 109.5 & $\mathrm{~N} 1-\mathrm{N} 2-\mathrm{C} 9$ & $117.31(11)$ \\
\hline $\mathrm{N} 2-\mathrm{C} 8-\mathrm{H} 8 \mathrm{~B}$ & 109.5 & $\mathrm{~N} 1-\mathrm{N} 2-\mathrm{C} 8$ & $121.56(10)$ \\
\hline $\mathrm{H} 8 \mathrm{~A}-\mathrm{C} 8-\mathrm{H} 8 \mathrm{~B}$ & 109.5 & $\mathrm{C} 9-\mathrm{N} 2-\mathrm{C} 8$ & $121.11(10)$ \\
\hline $\mathrm{N} 2-\mathrm{C} 8-\mathrm{H} 8 \mathrm{C}$ & 109.5 & $\mathrm{O} 3-\mathrm{N} 3-\mathrm{O} 2$ & $122.93(11)$ \\
\hline $\mathrm{H} 8 \mathrm{~A}-\mathrm{C} 8-\mathrm{H} 8 \mathrm{C}$ & 109.5 & $\mathrm{O} 3-\mathrm{N} 3-\mathrm{C} 3$ & $118.52(10)$ \\
\hline $\mathrm{H} 8 \mathrm{~B}-\mathrm{C} 8-\mathrm{H} 8 \mathrm{C}$ & 109.5 & $\mathrm{O} 2-\mathrm{N} 3-\mathrm{C} 3$ & $118.55(10)$ \\
\hline $\mathrm{O} 1-\mathrm{C} 9-\mathrm{N} 2$ & $121.03(12)$ & $\mathrm{C} 14-\mathrm{S} 1-\mathrm{C} 11$ & $92.35(6)$ \\
\hline $\mathrm{C} 6-\mathrm{C} 1-\mathrm{C} 2-\mathrm{C} 3$ & $0.08(18)$ & $\mathrm{S} 1-\mathrm{C} 11-\mathrm{C} 12-\mathrm{C} 13$ & $-0.83(15)$ \\
\hline $\mathrm{C} 7-\mathrm{C} 1-\mathrm{C} 2-\mathrm{C} 3$ & $-177.02(12)$ & $\mathrm{C} 11-\mathrm{C} 12-\mathrm{C} 13-\mathrm{C} 14$ & $0.49(18)$ \\
\hline $\mathrm{C} 1-\mathrm{C} 2-\mathrm{C} 3-\mathrm{C} 4$ & $1.15(19)$ & $\mathrm{C} 12-\mathrm{C} 13-\mathrm{C} 14-\mathrm{S} 1$ & $0.08(16)$ \\
\hline $\mathrm{C} 1-\mathrm{C} 2-\mathrm{C} 3-\mathrm{N} 3$ & $-178.86(11)$ & $\mathrm{C} 1-\mathrm{C} 7-\mathrm{N} 1-\mathrm{N} 2$ & $177.17(11)$ \\
\hline $\mathrm{C} 2-\mathrm{C} 3-\mathrm{C} 4-\mathrm{C} 5$ & $-1.5(2)$ & $\mathrm{C} 7-\mathrm{N} 1-\mathrm{N} 2-\mathrm{C} 9$ & $179.26(11)$ \\
\hline $\mathrm{N} 3-\mathrm{C} 3-\mathrm{C} 4-\mathrm{C} 5$ & $178.49(12)$ & $\mathrm{C} 7-\mathrm{N} 1-\mathrm{N} 2-\mathrm{C} 8$ & $-2.03(17)$ \\
\hline $\mathrm{C} 3-\mathrm{C} 4-\mathrm{C} 5-\mathrm{C} 6$ & $0.64(19)$ & $\mathrm{O} 1-\mathrm{C} 9-\mathrm{N} 2-\mathrm{N} 1$ & $179.67(11)$ \\
\hline $\mathrm{C} 4-\mathrm{C} 5-\mathrm{C} 6-\mathrm{C} 1$ & $0.5(2)$ & $\mathrm{C} 10-\mathrm{C} 9-\mathrm{N} 2-\mathrm{N} 1$ & $-0.78(16)$ \\
\hline $\mathrm{C} 2-\mathrm{C} 1-\mathrm{C} 6-\mathrm{C} 5$ & $-0.90(19)$ & $\mathrm{O} 1-\mathrm{C} 9-\mathrm{N} 2-\mathrm{C} 8$ & $0.95(19)$ \\
\hline $\mathrm{C} 7-\mathrm{C} 1-\mathrm{C} 6-\mathrm{C} 5$ & $176.30(12)$ & $\mathrm{C} 10-\mathrm{C} 9-\mathrm{N} 2-\mathrm{C} 8$ & $-179.50(11)$ \\
\hline $\mathrm{C} 2-\mathrm{C} 1-\mathrm{C} 7-\mathrm{N} 1$ & $5.17(19)$ & $\mathrm{C} 2-\mathrm{C} 3-\mathrm{N} 3-\mathrm{O} 3$ & $-1.60(18)$ \\
\hline $\mathrm{C} 6-\mathrm{C} 1-\mathrm{C} 7-\mathrm{N} 1$ & $-171.96(12)$ & $\mathrm{C} 4-\mathrm{C} 3-\mathrm{N} 3-\mathrm{O} 3$ & $178.39(12)$ \\
\hline $\mathrm{O} 1-\mathrm{C} 9-\mathrm{C} 10-\mathrm{C} 11$ & $-95.28(14)$ & $\mathrm{C} 2-\mathrm{C} 3-\mathrm{N} 3-\mathrm{O} 2$ & $178.30(11)$ \\
\hline $\mathrm{N} 2-\mathrm{C} 9-\mathrm{C} 10-\mathrm{C} 11$ & $85.18(14)$ & $\mathrm{C} 4-\mathrm{C} 3-\mathrm{N} 3-\mathrm{O} 2$ & $-1.70(17)$ \\
\hline $\mathrm{C} 9-\mathrm{C} 10-\mathrm{C} 11-\mathrm{C} 12$ & $108.80(15)$ & $\mathrm{C} 13-\mathrm{C} 14-\mathrm{S} 1-\mathrm{C} 11$ & $-0.47(12)$ \\
\hline $\mathrm{C} 9-\mathrm{C} 10-\mathrm{C} 11-\mathrm{S} 1$ & $-75.20(14)$ & $\mathrm{C} 12-\mathrm{C} 11-\mathrm{S} 1-\mathrm{C} 14$ & $0.74(11)$ \\
\hline $\mathrm{C} 10-\mathrm{C} 11-\mathrm{C} 12-\mathrm{C} 13$ & $175.57(13)$ & $\mathrm{C} 10-\mathrm{C} 11-\mathrm{S} 1-\mathrm{C} 14$ & $-175.84(11)$ \\
\hline
\end{tabular}


Hydrogen-bond geometry $\left(\AA,{ }^{\circ}\right)$

\begin{tabular}{lllll}
\hline$D-\mathrm{H} \cdots A$ & $D-\mathrm{H}$ & $\mathrm{H} \cdots A$ & $D \cdots A$ & $D-\mathrm{H} \cdots A$ \\
\hline $\mathrm{C} 7-\mathrm{H} 7 \cdots 2^{\mathrm{i}}$ & 0.95 & 2.39 & $3.2879(16)$ & 157 \\
$\mathrm{C} 8-\mathrm{H} 8 B^{\cdots} \cdots \mathrm{O} 2^{\mathrm{ii}}$ & 0.98 & 2.50 & $3.3468(16)$ & 144 \\
$\mathrm{C} 8-\mathrm{H} 8 C \cdots{ }^{\mathrm{iii}}$ & 0.98 & 2.52 & $3.4356(17)$ & 156 \\
$\mathrm{C} 13-\mathrm{H} 13 \cdots{ }^{\mathrm{ii}}$ & 0.95 & 2.52 & $3.1874(16)$ & 127 \\
\hline
\end{tabular}

Symmetry codes: (i) $-x+1 / 2, y-1 / 2,-z+1 / 2$; (ii) $x+1 / 2,-y+1 / 2, z+1 / 2$; (iii) $-x+3 / 2, y-1 / 2,-z+1 / 2$; (iv) $-x+2,-y+1,-z+1$.

(III) (E)-N-Methyl-N'-(4-nitrobenzylidene)-2-(thiophen-2-yl)acetohydrazide

\section{Crystal data}

$\mathrm{C}_{14} \mathrm{H}_{13} \mathrm{~N}_{3} \mathrm{O}_{3} \mathrm{~S}$

$M_{r}=303.33$

Triclinic, $P \overline{1}$

$a=6.1893(4) \AA$

$b=12.9177(9) \AA$

$c=17.3828(12) \AA$

$\alpha=93.995(7)^{\circ}$

$\beta=90.386(6)^{\circ}$

$\gamma=95.963(7)^{\circ}$

$V=1378.77(16) \AA^{3}$

\section{Data collection}

\section{Rigaku Mercury CCD}

diffractometer

$\omega$ scans

18534 measured reflections

6279 independent reflections

4868 reflections with $I>2 \sigma(I)$

\section{Refinement}

Refinement on $F^{2}$

Least-squares matrix: full

$R\left[F^{2}>2 \sigma\left(F^{2}\right)\right]=0.058$

$w R\left(F^{2}\right)=0.166$

$S=1.10$

6279 reflections

383 parameters

0 restraints

$$
\begin{aligned}
& Z=4 \\
& F(000)=632 \\
& D_{\mathrm{x}}=1.461 \mathrm{Mg} \mathrm{m}^{-3}
\end{aligned}
$$

Mo $K \alpha$ radiation, $\lambda=0.71073 \AA$

Cell parameters from 15464 reflections

$\theta=3.2-27.6^{\circ}$

$\mu=0.25 \mathrm{~mm}^{-1}$

$T=100 \mathrm{~K}$

Cut block, yellow

$0.20 \times 0.18 \times 0.16 \mathrm{~mm}$

Special details

Geometry. All esds (except the esd in the dihedral angle between two 1.s. planes) are estimated using the full covariance matrix. The cell esds are taken into account individually in the estimation of esds in distances, angles and torsion angles; correlations between esds in cell parameters are only used when they are defined by crystal symmetry. An approximate (isotropic) treatment of cell esds is used for estimating esds involving l.s. planes.

Fractional atomic coordinates and isotropic or equivalent isotropic displacement parameters $\left(\AA^{2}\right)$

\begin{tabular}{llllll}
\hline & $x$ & $y$ & $z$ & $U_{\text {iso }} * U_{\text {eq }}$ & Occ. $(<1)$ \\
\hline C1 & $0.3947(3)$ & $0.10043(14)$ & $0.12117(11)$ & $0.0207(4)$ & \\
C2 & $0.3601(3)$ & $0.01607(15)$ & $0.16786(12)$ & $0.0252(4)$ &
\end{tabular}




\begin{tabular}{|c|c|c|c|c|c|}
\hline $\mathrm{H} 2$ & 0.2397 & 0.0115 & 0.2015 & $0.030 *$ & \\
\hline $\mathrm{C} 3$ & $0.5005(4)$ & $-0.06029(15)$ & $0.16500(12)$ & $0.0271(5)$ & \\
\hline H3 & 0.4784 & -0.1176 & 0.1965 & $0.033 *$ & \\
\hline $\mathrm{C} 4$ & $0.6747(3)$ & $-0.05157(14)$ & $0.11517(12)$ & $0.0226(4)$ & \\
\hline C5 & $0.7135(3)$ & $0.03028(14)$ & $0.06866(11)$ & $0.0218(4)$ & \\
\hline H5 & 0.8345 & 0.0344 & 0.0353 & $0.026^{*}$ & \\
\hline C6 & $0.5712(3)$ & $0.10625(14)$ & $0.07199(11)$ & $0.0210(4)$ & \\
\hline H6 & 0.5945 & 0.1632 & 0.0402 & $0.025 *$ & \\
\hline $\mathrm{C} 7$ & $0.2465(3)$ & $0.18208(14)$ & $0.12183(11)$ & $0.0213(4)$ & \\
\hline $\mathrm{H} 7$ & 0.2660 & 0.2356 & 0.0869 & $0.026^{*}$ & \\
\hline $\mathrm{C} 8$ & $-0.0467(4)$ & $0.32516(15)$ & $0.10527(12)$ & $0.0255(4)$ & \\
\hline H8A & -0.0462 & 0.2846 & 0.0555 & $0.038^{*}$ & \\
\hline H8B & 0.0845 & 0.3748 & 0.1104 & $0.038 *$ & \\
\hline $\mathrm{H} 8 \mathrm{C}$ & -0.1755 & 0.3634 & 0.1081 & $0.038^{*}$ & \\
\hline C9 & $-0.2093(3)$ & $0.25364(14)$ & $0.22263(11)$ & $0.0220(4)$ & \\
\hline $\mathrm{C} 10$ & $-0.2139(3)$ & $0.17041(14)$ & $0.28040(12)$ & $0.0235(4)$ & \\
\hline $\mathrm{H} 10 \mathrm{~A}$ & -0.3660 & 0.1502 & 0.2950 & $0.028^{*}$ & \\
\hline H10B & -0.1553 & 0.1077 & 0.2562 & $0.028 *$ & \\
\hline C11 & -0.0820 & $0.20882(14)$ & $0.35164(11)$ & $0.0223(4)$ & \\
\hline $\mathrm{C} 12$ & $-0.1921(3)$ & $0.21642(9)$ & $0.43327(7)$ & $0.0459(5)$ & $0.673(3)$ \\
\hline H12 & -0.3381 & 0.1994 & 0.4483 & $0.055^{*}$ & $0.673(3)$ \\
\hline S1A & $-0.1921(3)$ & $0.21642(9)$ & $0.43327(7)$ & $0.0459(5)$ & $0.327(3)$ \\
\hline C13 & $0.0169(4)$ & $0.26112(17)$ & $0.48174(13)$ & $0.0325(5)$ & \\
\hline H13 & 0.0111 & 0.2762 & 0.5359 & $0.039 *$ & \\
\hline $\mathrm{C} 14$ & $0.2039(4)$ & $0.27710(16)$ & $0.44338(13)$ & $0.0318(5)$ & \\
\hline H14 & 0.3361 & 0.3048 & 0.4687 & $0.038 *$ & \\
\hline N1 & $0.0911(3)$ & $0.18039(12)$ & $0.16982(9)$ & $0.0207(4)$ & \\
\hline $\mathrm{N} 2$ & $-0.0509(3)$ & $0.25508(12)$ & $0.16718(10)$ & $0.0214(4)$ & \\
\hline N3 & $0.8213(3)$ & -0.13383 & $0.11193(11)$ & $0.0282(4)$ & \\
\hline $\mathrm{O} 1$ & $-0.3402(2)$ & $0.31816(11)$ & $0.22537(9)$ & 0.0288 & \\
\hline $\mathrm{O} 2$ & $0.9584(2)$ & $-0.13473(11)$ & $0.06134(9)$ & $0.0323(4)$ & \\
\hline $\mathrm{O} 3$ & $0.7993(3)$ & $-0.19851(13)$ & $0.16061(11)$ & $0.0489(5)$ & \\
\hline S1 & $0.18984(12)$ & $0.24543(5)$ & $0.34894(4)$ & $0.0275(2)$ & $0.673(3)$ \\
\hline $\mathrm{C} 12 \mathrm{~A}$ & $0.18984(12)$ & $0.24543(5)$ & $0.34894(4)$ & $0.0275(2)$ & $0.327(3)$ \\
\hline $\mathrm{H} 12 \mathrm{~A}$ & 0.2936 & 0.2471 & 0.3089 & $0.033^{*}$ & $0.327(3)$ \\
\hline $\mathrm{C} 15$ & $-0.1307(3)$ & $0.45425(14)$ & $0.76584(11)$ & $0.0204(4)$ & \\
\hline $\mathrm{C} 16$ & $-0.3149(3)$ & $0.50824(13)$ & $0.77270(11)$ & $0.0211(4)$ & \\
\hline H16 & -0.3395 & 0.5585 & 0.7371 & $0.025^{*}$ & \\
\hline C17 & -0.4611 & $0.48896(14)$ & $0.83085(11)$ & $0.0213(4)$ & \\
\hline H17 & -0.5869 & 0.5252 & 0.8355 & $0.026^{*}$ & \\
\hline C18 & -0.4209 & $0.41575(14)$ & $0.88233(11)$ & $0.0215(4)$ & \\
\hline C19 & -0.2380 & $0.36200(15)$ & $0.87768(12)$ & $0.0237(4)$ & \\
\hline H19 & -0.2135 & 0.3122 & 0.9136 & $0.028 *$ & \\
\hline $\mathrm{C} 20$ & -0.0932 & $0.38266(14)$ & $0.81973(12)$ & 0.0228 & \\
\hline $\mathrm{H} 20$ & 0.0344 & 0.3477 & 0.8163 & $0.027^{*}$ & \\
\hline $\mathrm{C} 21$ & $0.0221(3)$ & $0.47259(14)$ & $0.70286(11)$ & $0.0211(4)$ & \\
\hline $\mathrm{H} 21$ & 0.0129 & 0.5292 & 0.6713 & $0.025 *$ & \\
\hline $\mathrm{C} 22$ & $0.3281(4)$ & $0.52050(16)$ & $0.59201(12)$ & $0.0302(5)$ & \\
\hline
\end{tabular}




\begin{tabular}{|c|c|c|c|c|c|}
\hline $\mathrm{H} 22 \mathrm{~A}$ & 0.3299 & 0.5823 & 0.6281 & $0.045^{*}$ & \\
\hline $\mathrm{H} 22 \mathrm{~B}$ & 0.2012 & 0.5167 & 0.5575 & $0.045^{*}$ & \\
\hline $\mathrm{H} 22 \mathrm{C}$ & 0.4609 & 0.5252 & 0.5615 & $0.045^{*}$ & \\
\hline C23 & $0.4675(3)$ & 0.35588 (14) & $0.62403(11)$ & $0.0216(4)$ & \\
\hline $\mathrm{C} 24$ & $0.4422(3)$ & $0.26350(14)$ & $0.67397(12)$ & $0.0235(4)$ & \\
\hline $\mathrm{H} 24 \mathrm{~A}$ & 0.4741 & 0.2890 & 0.7283 & $0.028 *$ & \\
\hline H24B & 0.2893 & 0.2322 & 0.6708 & $0.028^{*}$ & \\
\hline $\mathrm{C} 25$ & 0.5867 (4) & 0.18068 (14) & $0.65165(12)$ & $0.0247(4)$ & \\
\hline $\mathrm{C} 26$ & $0.8082(3)$ & $0.17391(11)$ & 0.67653 (9) & $0.0332(5)$ & $0.832(3)$ \\
\hline H26 & 0.8933 & 0.2246 & 0.7093 & $0.040 *$ & $0.832(3)$ \\
\hline S2A & $0.8082(3)$ & $0.17391(11)$ & $0.67653(9)$ & $0.0332(5)$ & 0.168 (3) \\
\hline $\mathrm{C} 27$ & 0.8783 (4) & $0.07846(18)$ & $0.64370(14)$ & $0.0363(5)$ & \\
\hline H27 & 1.0182 & 0.0579 & 0.6536 & $0.044 *$ & \\
\hline $\mathrm{C} 28$ & $0.7311(5)$ & $0.02082(17)$ & $0.59807(15)$ & $0.0452(7)$ & \\
\hline H28 & 0.7552 & -0.0441 & 0.5722 & $0.054 *$ & \\
\hline N4 & 0.1678 (3) & $0.41024(12)$ & $0.69164(9)$ & $0.0204(4)$ & \\
\hline N5 & $0.3168(3)$ & 0.42739 (12) & $0.63490(9)$ & 0.0218 (4) & \\
\hline N6 & $-0.5754(3)$ & $0.39473(13)$ & $0.94419(10)$ & 0.0249 (4) & \\
\hline $\mathrm{O} 4$ & $0.6102(2)$ & $0.36793(11)$ & $0.57669(8)$ & $0.0279(3)$ & \\
\hline $\mathrm{O} 5$ & $-0.7216(2)$ & 0.45094 (11) & $0.95462(9)$ & $0.0296(3)$ & \\
\hline O6 & $-0.5498(3)$ & $0.32116(12)$ & $0.98361(10)$ & $0.0373(4)$ & \\
\hline S2 & $0.49917(12)$ & $0.07608(5)$ & 0.59164 (4) & $0.0380(3)$ & $0.832(3)$ \\
\hline $\mathrm{C} 26 \mathrm{~A}$ & $0.49917(12)$ & $0.07608(5)$ & $0.59164(4)$ & $0.0380(3)$ & 0.168 (3) \\
\hline $\mathrm{H} 26 \mathrm{~A}$ & 0.3665 & 0.0559 & 0.5642 & $0.046^{*}$ & $0.168(3)$ \\
\hline
\end{tabular}

Atomic displacement parameters $\left(\AA^{2}\right)$

\begin{tabular}{lllllll}
\hline & $U^{11}$ & $U^{22}$ & $U^{33}$ & $U^{12}$ & $U^{13}$ & $U^{23}$ \\
\hline C1 & $0.0237(10)$ & $0.0193(8)$ & $0.0186(9)$ & $0.0028(7)$ & $-0.0027(8)$ & $-0.0026(7)$ \\
C2 & $0.0270(11)$ & $0.0258(9)$ & $0.0235(11)$ & $0.0057(8)$ & $0.0055(9)$ & $0.0022(8)$ \\
C3 & $0.0359(12)$ & $0.0220(9)$ & $0.0246(11)$ & $0.0063(8)$ & $0.0024(9)$ & $0.0041(8)$ \\
C4 & $0.0258(10)$ & $0.0205(9)$ & $0.0218(10)$ & $0.0078(8)$ & $-0.0022(8)$ & $-0.0041(7)$ \\
C5 & $0.0230(10)$ & $0.0210(9)$ & $0.0204(10)$ & $0.0014(7)$ & $-0.0008(8)$ & $-0.0040(7)$ \\
C6 & $0.0253(10)$ & $0.0167(8)$ & $0.0202(10)$ & $0.0009(7)$ & $-0.0023(8)$ & $-0.0014(7)$ \\
C7 & $0.0246(10)$ & $0.0188(8)$ & $0.0203(10)$ & $0.0017(7)$ & $-0.0008(8)$ & $0.0004(7)$ \\
C8 & $0.0341(12)$ & $0.0223(9)$ & $0.0207(10)$ & $0.0079(8)$ & $-0.0023(9)$ & $-0.0008(8)$ \\
C9 & $0.0240(10)$ & $0.0216(9)$ & $0.0197(10)$ & $0.0035(8)$ & $-0.0025(8)$ & $-0.0049(7)$ \\
C10 & $0.0255(10)$ & $0.0202(9)$ & $0.0245(11)$ & $0.0035(8)$ & $0.0015(9)$ & $-0.0025(8)$ \\
C11 & $0.0297(11)$ & $0.0175(8)$ & $0.0203(10)$ & $0.0056(8)$ & $0.0037(8)$ & $0.0012(7)$ \\
C12 & $0.0713(10)$ & $0.0336(7)$ & $0.0324(7)$ & $0.0083(6)$ & $-0.0152(7)$ & $-0.0034(5)$ \\
S1A & $0.0713(10)$ & $0.0336(7)$ & $0.0324(7)$ & $0.0083(6)$ & $-0.0152(7)$ & $-0.0034(5)$ \\
C13 & $0.0431(13)$ & $0.0343(11)$ & $0.0211(11)$ & $0.0075(10)$ & $0.0028(10)$ & $0.0031(9)$ \\
C14 & $0.0384(13)$ & $0.0269(10)$ & $0.0296(12)$ & $0.0031(9)$ & $0.0000(10)$ & $-0.0002(9)$ \\
N1 & $0.0234(9)$ & $0.0196(7)$ & $0.0191(8)$ & $0.0052(6)$ & $-0.0032(7)$ & $-0.0040(6)$ \\
N2 & $0.0245(9)$ & $0.0209(7)$ & $0.0193(8)$ & $0.0065(6)$ & $-0.0011(7)$ & $-0.0016(6)$ \\
N3 & $0.0332(10)$ & $0.0259(8)$ & $0.0266(10)$ & $0.0110(7)$ & $0.0013(8)$ & $-0.0009(7)$ \\
O1 & $0.0329(8)$ & $0.0276(7)$ & $0.0272(8)$ & $0.0124(6)$ & $0.0013(7)$ & $-0.0037(6)$ \\
O2 & $0.0324(9)$ & $0.0300(7)$ & $0.0359(9)$ & $0.0111(6)$ & $0.0100(7)$ & $-0.0013(7)$
\end{tabular}




\begin{tabular}{lllllll}
\hline O3 & $0.0667(13)$ & $0.0449(10)$ & $0.0436(11)$ & $0.0335(9)$ & $0.0174(9)$ & $0.0205(8)$ \\
S1 & $0.0339(4)$ & $0.0253(4)$ & $0.0227(4)$ & $0.0008(3)$ & $-0.0028(3)$ & $0.0004(3)$ \\
C12A & $0.0339(4)$ & $0.0253(4)$ & $0.0227(4)$ & $0.0008(3)$ & $-0.0028(3)$ & $0.0004(3)$ \\
C15 & $0.0249(10)$ & $0.0169(8)$ & $0.0184(10)$ & $0.0020(7)$ & $-0.0025(8)$ & $-0.0046(7)$ \\
C16 & $0.0288(10)$ & $0.0152(8)$ & $0.0194(10)$ & $0.0040(7)$ & $-0.0029(8)$ & $-0.0002(7)$ \\
C17 & $0.0243(10)$ & $0.0190(8)$ & $0.0206(10)$ & $0.0051(7)$ & $-0.0019(8)$ & $-0.0038(7)$ \\
C18 & $0.0262(10)$ & $0.0197(8)$ & $0.0178(10)$ & $0.0021(8)$ & $0.0016(8)$ & $-0.0043(7)$ \\
C19 & $0.0304(11)$ & $0.0232(9)$ & $0.0186(10)$ & $0.0076(8)$ & $0.0003(8)$ & $0.0011(7)$ \\
C20 & $0.0252(10)$ & $0.0227(9)$ & $0.0214(10)$ & $0.0091(8)$ & $0.0005(8)$ & $-0.0026(7)$ \\
C21 & $0.0275(11)$ & $0.0184(8)$ & $0.0171(9)$ & $0.0032(7)$ & $-0.0017(8)$ & $-0.0003(7)$ \\
C22 & $0.0442(13)$ & $0.0246(10)$ & $0.0232(11)$ & $0.0080(9)$ & $0.0080(10)$ & $0.0046(8)$ \\
C23 & $0.0264(11)$ & $0.0205(9)$ & $0.0167(9)$ & $0.0013(8)$ & $-0.0009(8)$ & $-0.0040(7)$ \\
C24 & $0.0294(11)$ & $0.0211(9)$ & $0.0203(10)$ & $0.0044(8)$ & $0.0043(8)$ & $0.0000(7)$ \\
C25 & $0.0356(12)$ & $0.0198(9)$ & $0.0190(10)$ & $0.0045(8)$ & $0.0075(9)$ & $0.0000(7)$ \\
C26 & $0.0387(10)$ & $0.0257(7)$ & $0.0347(9)$ & $0.0027(6)$ & $0.0045(7)$ & $-0.0006(6)$ \\
S2A & $0.0387(10)$ & $0.0257(7)$ & $0.0347(9)$ & $0.0027(6)$ & $0.0045(7)$ & $-0.0006(6)$ \\
C27 & $0.0370(13)$ & $0.0357(12)$ & $0.0393(14)$ & $0.0140(10)$ & $0.0097(11)$ & $0.0088(10)$ \\
C28 & $0.079(2)$ & $0.0235(10)$ & $0.0347(14)$ & $0.0187(12)$ & $0.0072(13)$ & $-0.0043(9)$ \\
N4 & $0.0248(9)$ & $0.0193(7)$ & $0.0160(8)$ & $0.0004(6)$ & $0.0015(7)$ & $-0.0036(6)$ \\
N5 & $0.0284(9)$ & $0.0202(7)$ & $0.0167(8)$ & $0.0032(7)$ & $0.0041(7)$ & $-0.0002(6)$ \\
N6 & $0.0270(9)$ & $0.0254(8)$ & $0.0218(9)$ & $0.0028(7)$ & $0.0010(7)$ & $-0.0011(7)$ \\
O4 & $0.0335(8)$ & $0.0285(7)$ & $0.0224(8)$ & $0.0056(6)$ & $0.0096(7)$ & $0.0022(6)$ \\
O5 & $0.0285(8)$ & $0.0346(8)$ & $0.0266(8)$ & $0.0100(6)$ & $0.0057(6)$ & $-0.0021(6)$ \\
O6 & $0.0451(10)$ & $0.0363(8)$ & $0.0334(9)$ & $0.0107(7)$ & $0.0114(8)$ & $0.0138(7)$ \\
S2 & $0.0456(5)$ & $0.0264(3)$ & $0.0403(4)$ & $0.0086(3)$ & $-0.0115(3)$ & $-0.0154(3)$ \\
C26A & $0.0456(5)$ & $0.0264(3)$ & $0.0403(4)$ & $0.0086(3)$ & $-0.0115(3)$ & $-0.0154(3)$ \\
& & & & & & \\
\hline & & & & & & \\
\hline
\end{tabular}

Geometric parameters $\left(A,{ }^{o}\right)$

\begin{tabular}{llll}
\hline $\mathrm{C} 1-\mathrm{C} 6$ & $1.391(3)$ & $\mathrm{C} 15-\mathrm{C} 20$ & $1.396(3)$ \\
$\mathrm{C} 1-\mathrm{C} 2$ & $1.403(3)$ & $\mathrm{C} 15-\mathrm{C} 16$ & $1.398(3)$ \\
$\mathrm{C} 1-\mathrm{C} 7$ & $1.467(3)$ & $\mathrm{C} 15-\mathrm{C} 21$ & $1.467(3)$ \\
$\mathrm{C} 2-\mathrm{C} 3$ & $1.379(3)$ & $\mathrm{C} 16-\mathrm{C} 17$ & $1.380(3)$ \\
$\mathrm{C} 2-\mathrm{H} 2$ & 0.9500 & $\mathrm{C} 16-\mathrm{H} 16$ & 0.9500 \\
$\mathrm{C} 3-\mathrm{C} 4$ & $1.388(3)$ & $\mathrm{C} 17-\mathrm{C} 18$ & $1.386(3)$ \\
$\mathrm{C} 3-\mathrm{H} 3$ & 0.9500 & $\mathrm{C} 17-\mathrm{H} 17$ & 0.9500 \\
$\mathrm{C} 4-\mathrm{C} 5$ & $1.378(3)$ & $\mathrm{C} 18-\mathrm{C} 19$ & $1.388(3)$ \\
$\mathrm{C} 4-\mathrm{N} 3$ & $1.466(2)$ & $\mathrm{C} 18-\mathrm{N} 6$ & $1.464(3)$ \\
$\mathrm{C} 5-\mathrm{C} 6$ & $1.384(3)$ & $\mathrm{C} 19-\mathrm{C} 20$ & $1.375(3)$ \\
$\mathrm{C} 5-\mathrm{H} 5$ & 0.9500 & $\mathrm{C} 19-\mathrm{H} 19$ & 0.9500 \\
$\mathrm{C} 6-\mathrm{H} 6$ & 0.9500 & $\mathrm{C} 20-\mathrm{H} 20$ & 0.9500 \\
$\mathrm{C} 7-\mathrm{N} 1$ & $1.277(3)$ & $\mathrm{C} 21-\mathrm{N} 4$ & $1.277(2)$ \\
$\mathrm{C} 7-\mathrm{H} 7$ & 0.9500 & $\mathrm{C} 21-\mathrm{H} 21$ & 0.9500 \\
$\mathrm{C} 8-\mathrm{N} 2$ & $1.452(3)$ & $\mathrm{C} 22-\mathrm{N} 5$ & $1.454(2)$ \\
$\mathrm{C} 8-\mathrm{H} 8 \mathrm{~A}$ & 0.9800 & $\mathrm{C} 22-\mathrm{H} 22 \mathrm{~A}$ & 0.9800 \\
$\mathrm{C} 8-\mathrm{H} 8 \mathrm{~B}$ & 0.9800 & $\mathrm{C} 22-\mathrm{H} 22 \mathrm{~B}$ & 0.9800 \\
$\mathrm{C} 8-\mathrm{H} 8 \mathrm{C}$ & 0.9800 & $\mathrm{C} 22-\mathrm{H} 22 \mathrm{C}$ & 0.9800 \\
$\mathrm{C} 9-\mathrm{O} 1$ & $1.220(2)$ & $\mathrm{C} 23-\mathrm{O} 4$ & $1.217(2)$
\end{tabular}




\begin{tabular}{|c|c|c|c|}
\hline $\mathrm{C} 9-\mathrm{N} 2$ & $1.380(3)$ & $\mathrm{C} 23-\mathrm{N} 5$ & $1.384(2)$ \\
\hline $\mathrm{C} 9-\mathrm{C} 10$ & $1.520(3)$ & $\mathrm{C} 23-\mathrm{C} 24$ & $1.520(3)$ \\
\hline $\mathrm{C} 10-\mathrm{C} 11$ & $1.504(3)$ & $\mathrm{C} 24-\mathrm{C} 25$ & $1.497(3)$ \\
\hline $\mathrm{C} 10-\mathrm{H} 10 \mathrm{~A}$ & 0.9900 & $\mathrm{C} 24-\mathrm{H} 24 \mathrm{~A}$ & 0.9900 \\
\hline $\mathrm{C} 10-\mathrm{H} 10 \mathrm{~B}$ & 0.9900 & $\mathrm{C} 24-\mathrm{H} 24 \mathrm{~B}$ & 0.9900 \\
\hline $\mathrm{C} 11-\mathrm{S} 1 \mathrm{~A}$ & $1.579(2)$ & $\mathrm{C} 25-\mathrm{S} 2 \mathrm{~A}$ & $1.448(3)$ \\
\hline $\mathrm{C} 11-\mathrm{C} 12$ & $1.579(2)$ & $\mathrm{C} 25-\mathrm{C} 26$ & $1.448(3)$ \\
\hline $\mathrm{C} 11-\mathrm{C} 12 \mathrm{~A}$ & $1.702(2)$ & $\mathrm{C} 25-\mathrm{C} 26 \mathrm{~A}$ & $1.689(2)$ \\
\hline $\mathrm{C} 11-\mathrm{S} 1$ & $1.702(2)$ & $\mathrm{C} 25-\mathrm{S} 2$ & $1.689(2)$ \\
\hline $\mathrm{C} 12-\mathrm{C} 13$ & $1.575(3)$ & $\mathrm{C} 26-\mathrm{C} 27$ & $1.432(3)$ \\
\hline $\mathrm{C} 12-\mathrm{H} 12$ & 0.9500 & $\mathrm{C} 26-\mathrm{H} 26$ & 0.9500 \\
\hline $\mathrm{S} 1 \mathrm{~A}-\mathrm{C} 13$ & $1.575(3)$ & $\mathrm{S} 2 \mathrm{~A}-\mathrm{C} 27$ & $1.432(3)$ \\
\hline $\mathrm{C} 13-\mathrm{C} 14$ & $1.344(3)$ & $\mathrm{C} 27-\mathrm{C} 28$ & $1.334(4)$ \\
\hline C13-H13 & 0.9500 & $\mathrm{C} 27-\mathrm{H} 27$ & 0.9500 \\
\hline $\mathrm{C} 14-\mathrm{C} 12 \mathrm{~A}$ & $1.663(2)$ & $\mathrm{C} 28-\mathrm{C} 26 \mathrm{~A}$ & $1.675(3)$ \\
\hline $\mathrm{C} 14-\mathrm{S} 1$ & $1.663(2)$ & $\mathrm{C} 28-\mathrm{S} 2$ & $1.675(3)$ \\
\hline C14-H14 & 0.9500 & $\mathrm{C} 28-\mathrm{H} 28$ & 0.9500 \\
\hline $\mathrm{N} 1-\mathrm{N} 2$ & $1.373(2)$ & $\mathrm{N} 4-\mathrm{N} 5$ & $1.368(2)$ \\
\hline $\mathrm{N} 3-\mathrm{O} 2$ & $1.227(2)$ & $\mathrm{N} 6-\mathrm{O} 5$ & $1.224(2)$ \\
\hline $\mathrm{N} 3-\mathrm{O} 3$ & $1.228(2)$ & $\mathrm{N} 6-\mathrm{O} 6$ & $1.232(2)$ \\
\hline $\mathrm{C} 12 \mathrm{~A}-\mathrm{H} 12 \mathrm{~A}$ & 0.9500 & $\mathrm{C} 26 \mathrm{~A}-\mathrm{H} 26 \mathrm{~A}$ & 0.9500 \\
\hline $\mathrm{C} 6-\mathrm{C} 1-\mathrm{C} 2$ & $119.49(18)$ & $\mathrm{C} 20-\mathrm{C} 15-\mathrm{C} 16$ & $119.30(18)$ \\
\hline $\mathrm{C} 6-\mathrm{C} 1-\mathrm{C} 7$ & $118.95(17)$ & $\mathrm{C} 20-\mathrm{C} 15-\mathrm{C} 21$ & $120.19(18)$ \\
\hline $\mathrm{C} 2-\mathrm{C} 1-\mathrm{C} 7$ & $121.55(18)$ & $\mathrm{C} 16-\mathrm{C} 15-\mathrm{C} 21$ & $120.51(17)$ \\
\hline $\mathrm{C} 3-\mathrm{C} 2-\mathrm{C} 1$ & $120.15(19)$ & $\mathrm{C} 17-\mathrm{C} 16-\mathrm{C} 15$ & $120.39(17)$ \\
\hline $\mathrm{C} 3-\mathrm{C} 2-\mathrm{H} 2$ & 119.9 & $\mathrm{C} 17-\mathrm{C} 16-\mathrm{H} 16$ & 119.8 \\
\hline $\mathrm{C} 1-\mathrm{C} 2-\mathrm{H} 2$ & 119.9 & $\mathrm{C} 15-\mathrm{C} 16-\mathrm{H} 16$ & 119.8 \\
\hline $\mathrm{C} 2-\mathrm{C} 3-\mathrm{C} 4$ & $118.57(18)$ & $\mathrm{C} 16-\mathrm{C} 17-\mathrm{C} 18$ & $118.71(18)$ \\
\hline $\mathrm{C} 2-\mathrm{C} 3-\mathrm{H} 3$ & 120.7 & $\mathrm{C} 16-\mathrm{C} 17-\mathrm{H} 17$ & 120.6 \\
\hline $\mathrm{C} 4-\mathrm{C} 3-\mathrm{H} 3$ & 120.7 & $\mathrm{C} 18-\mathrm{C} 17-\mathrm{H} 17$ & 120.6 \\
\hline $\mathrm{C} 5-\mathrm{C} 4-\mathrm{C} 3$ & $122.75(18)$ & $\mathrm{C} 17-\mathrm{C} 18-\mathrm{C} 19$ & $122.23(18)$ \\
\hline $\mathrm{C} 5-\mathrm{C} 4-\mathrm{N} 3$ & $119.17(18)$ & $\mathrm{C} 17-\mathrm{C} 18-\mathrm{N} 6$ & $119.07(17)$ \\
\hline $\mathrm{C} 3-\mathrm{C} 4-\mathrm{N} 3$ & $118.08(17)$ & $\mathrm{C} 19-\mathrm{C} 18-\mathrm{N} 6$ & $118.70(17)$ \\
\hline $\mathrm{C} 4-\mathrm{C} 5-\mathrm{C} 6$ & $118.10(18)$ & $\mathrm{C} 20-\mathrm{C} 19-\mathrm{C} 18$ & $118.32(18)$ \\
\hline $\mathrm{C} 4-\mathrm{C} 5-\mathrm{H} 5$ & 121.0 & $\mathrm{C} 20-\mathrm{C} 19-\mathrm{H} 19$ & 120.8 \\
\hline $\mathrm{C} 6-\mathrm{C} 5-\mathrm{H} 5$ & 121.0 & $\mathrm{C} 18-\mathrm{C} 19-\mathrm{H} 19$ & 120.8 \\
\hline $\mathrm{C} 5-\mathrm{C} 6-\mathrm{C} 1$ & $120.95(17)$ & $\mathrm{C} 19-\mathrm{C} 20-\mathrm{C} 15$ & $121.02(18)$ \\
\hline $\mathrm{C} 5-\mathrm{C} 6-\mathrm{H} 6$ & 119.5 & $\mathrm{C} 19-\mathrm{C} 20-\mathrm{H} 20$ & 119.5 \\
\hline $\mathrm{C} 1-\mathrm{C} 6-\mathrm{H} 6$ & 119.5 & $\mathrm{C} 15-\mathrm{C} 20-\mathrm{H} 20$ & 119.5 \\
\hline $\mathrm{N} 1-\mathrm{C} 7-\mathrm{C} 1$ & $119.28(17)$ & $\mathrm{N} 4-\mathrm{C} 21-\mathrm{C} 15$ & $118.09(17)$ \\
\hline $\mathrm{N} 1-\mathrm{C} 7-\mathrm{H} 7$ & 120.4 & $\mathrm{~N} 4-\mathrm{C} 21-\mathrm{H} 21$ & 121.0 \\
\hline $\mathrm{C} 1-\mathrm{C} 7-\mathrm{H} 7$ & 120.4 & $\mathrm{C} 15-\mathrm{C} 21-\mathrm{H} 21$ & 121.0 \\
\hline $\mathrm{N} 2-\mathrm{C} 8-\mathrm{H} 8 \mathrm{~A}$ & 109.5 & $\mathrm{~N} 5-\mathrm{C} 22-\mathrm{H} 22 \mathrm{~A}$ & 109.5 \\
\hline $\mathrm{N} 2-\mathrm{C} 8-\mathrm{H} 8 \mathrm{~B}$ & 109.5 & $\mathrm{~N} 5-\mathrm{C} 22-\mathrm{H} 22 \mathrm{~B}$ & 109.5 \\
\hline $\mathrm{H} 8 \mathrm{~A}-\mathrm{C} 8-\mathrm{H} 8 \mathrm{~B}$ & 109.5 & $\mathrm{H} 22 \mathrm{~A}-\mathrm{C} 22-\mathrm{H} 22 \mathrm{~B}$ & 109.5 \\
\hline $\mathrm{N} 2-\mathrm{C} 8-\mathrm{H} 8 \mathrm{C}$ & 109.5 & $\mathrm{~N} 5-\mathrm{C} 22-\mathrm{H} 22 \mathrm{C}$ & 109.5 \\
\hline $\mathrm{H} 8 \mathrm{~A}-\mathrm{C} 8-\mathrm{H} 8 \mathrm{C}$ & 109.5 & $\mathrm{H} 22 \mathrm{~A}-\mathrm{C} 22-\mathrm{H} 22 \mathrm{C}$ & 109.5 \\
\hline
\end{tabular}




\begin{tabular}{|c|c|c|c|}
\hline $\mathrm{H} 8 \mathrm{~B}-\mathrm{C} 8-\mathrm{H} 8 \mathrm{C}$ & 109.5 & $\mathrm{H} 22 \mathrm{~B}-\mathrm{C} 22-\mathrm{H} 22 \mathrm{C}$ & 109.5 \\
\hline $\mathrm{O} 1-\mathrm{C} 9-\mathrm{N} 2$ & $120.65(18)$ & $\mathrm{O} 4-\mathrm{C} 23-\mathrm{N} 5$ & $120.73(17)$ \\
\hline $\mathrm{O} 1-\mathrm{C} 9-\mathrm{C} 10$ & $121.28(18)$ & $\mathrm{O} 4-\mathrm{C} 23-\mathrm{C} 24$ & $123.10(17)$ \\
\hline $\mathrm{N} 2-\mathrm{C} 9-\mathrm{C} 10$ & $118.07(16)$ & $\mathrm{N} 5-\mathrm{C} 23-\mathrm{C} 24$ & $116.17(17)$ \\
\hline $\mathrm{C} 11-\mathrm{C} 10-\mathrm{C} 9$ & $111.36(15)$ & $\mathrm{C} 25-\mathrm{C} 24-\mathrm{C} 23$ & $113.87(17)$ \\
\hline $\mathrm{C} 11-\mathrm{C} 10-\mathrm{H} 10 \mathrm{~A}$ & 109.4 & $\mathrm{C} 25-\mathrm{C} 24-\mathrm{H} 24 \mathrm{~A}$ & 108.8 \\
\hline $\mathrm{C} 9-\mathrm{C} 10-\mathrm{H} 10 \mathrm{~A}$ & 109.4 & $\mathrm{C} 23-\mathrm{C} 24-\mathrm{H} 24 \mathrm{~A}$ & 108.8 \\
\hline $\mathrm{C} 11-\mathrm{C} 10-\mathrm{H} 10 \mathrm{~B}$ & 109.4 & $\mathrm{C} 25-\mathrm{C} 24-\mathrm{H} 24 \mathrm{~B}$ & 108.8 \\
\hline $\mathrm{C} 9-\mathrm{C} 10-\mathrm{H} 10 \mathrm{~B}$ & 109.4 & $\mathrm{C} 23-\mathrm{C} 24-\mathrm{H} 24 \mathrm{~B}$ & 108.8 \\
\hline $\mathrm{H} 10 \mathrm{~A}-\mathrm{C} 10-\mathrm{H} 10 \mathrm{~B}$ & 108.0 & $\mathrm{H} 24 \mathrm{~A}-\mathrm{C} 24-\mathrm{H} 24 \mathrm{~B}$ & 107.7 \\
\hline $\mathrm{C} 10-\mathrm{C} 11-\mathrm{S} 1 \mathrm{~A}$ & $120.81(16)$ & $\mathrm{S} 2 \mathrm{~A}-\mathrm{C} 25-\mathrm{C} 24$ & $128.30(17)$ \\
\hline $\mathrm{C} 10-\mathrm{C} 11-\mathrm{C} 12$ & $120.81(16)$ & $\mathrm{C} 26-\mathrm{C} 25-\mathrm{C} 24$ & $128.30(17)$ \\
\hline $\mathrm{C} 10-\mathrm{C} 11-\mathrm{C} 12 \mathrm{~A}$ & $122.36(15)$ & $\mathrm{S} 2 \mathrm{~A}-\mathrm{C} 25-\mathrm{C} 26 \mathrm{~A}$ & $110.16(13)$ \\
\hline $\mathrm{S} 1 \mathrm{~A}-\mathrm{C} 11-\mathrm{C} 12 \mathrm{~A}$ & $116.83(13)$ & $\mathrm{C} 24-\mathrm{C} 25-\mathrm{C} 26 \mathrm{~A}$ & $121.52(16)$ \\
\hline $\mathrm{C} 10-\mathrm{C} 11-\mathrm{S} 1$ & $122.36(15)$ & $\mathrm{C} 26-\mathrm{C} 25-\mathrm{S} 2$ & $110.16(13)$ \\
\hline $\mathrm{C} 12-\mathrm{C} 11-\mathrm{S} 1$ & $116.83(13)$ & $\mathrm{C} 24-\mathrm{C} 25-\mathrm{S} 2$ & $121.52(16)$ \\
\hline $\mathrm{C} 13-\mathrm{C} 12-\mathrm{C} 11$ & $97.55(14)$ & $\mathrm{C} 27-\mathrm{C} 26-\mathrm{C} 25$ & $108.93(16)$ \\
\hline $\mathrm{C} 13-\mathrm{C} 12-\mathrm{H} 12$ & 131.2 & $\mathrm{C} 27-\mathrm{C} 26-\mathrm{H} 26$ & 125.5 \\
\hline $\mathrm{C} 11-\mathrm{C} 12-\mathrm{H} 12$ & 131.2 & $\mathrm{C} 25-\mathrm{C} 26-\mathrm{H} 26$ & 125.5 \\
\hline $\mathrm{C} 13-\mathrm{S} 1 \mathrm{~A}-\mathrm{C} 11$ & $97.55(14)$ & $\mathrm{C} 27-\mathrm{S} 2 \mathrm{~A}-\mathrm{C} 25$ & $108.93(16)$ \\
\hline $\mathrm{C} 14-\mathrm{C} 13-\mathrm{C} 12$ & $117.32(19)$ & $\mathrm{C} 28-\mathrm{C} 27-\mathrm{C} 26$ & $114.6(2)$ \\
\hline $\mathrm{C} 14-\mathrm{C} 13-\mathrm{S} 1 \mathrm{~A}$ & $117.32(19)$ & $\mathrm{C} 28-\mathrm{C} 27-\mathrm{S} 2 \mathrm{~A}$ & $114.6(2)$ \\
\hline $\mathrm{C} 14-\mathrm{C} 13-\mathrm{H} 13$ & 121.3 & $\mathrm{C} 28-\mathrm{C} 27-\mathrm{H} 27$ & 122.7 \\
\hline $\mathrm{C} 12-\mathrm{C} 13-\mathrm{H} 13$ & 121.3 & $\mathrm{C} 26-\mathrm{C} 27-\mathrm{H} 27$ & 122.7 \\
\hline $\mathrm{C} 13-\mathrm{C} 14-\mathrm{C} 12 \mathrm{~A}$ & $115.93(18)$ & $\mathrm{C} 27-\mathrm{C} 28-\mathrm{C} 26 \mathrm{~A}$ & $112.26(17)$ \\
\hline $\mathrm{C} 13-\mathrm{C} 14-\mathrm{S} 1$ & $115.93(18)$ & $\mathrm{C} 27-\mathrm{C} 28-\mathrm{S} 2$ & $112.26(17)$ \\
\hline $\mathrm{C} 13-\mathrm{C} 14-\mathrm{H} 14$ & 122.0 & $\mathrm{C} 27-\mathrm{C} 28-\mathrm{H} 28$ & 123.9 \\
\hline $\mathrm{S} 1-\mathrm{C} 14-\mathrm{H} 14$ & 122.0 & $\mathrm{~S} 2-\mathrm{C} 28-\mathrm{H} 28$ & 123.9 \\
\hline $\mathrm{C} 7-\mathrm{N} 1-\mathrm{N} 2$ & $118.20(16)$ & $\mathrm{C} 21-\mathrm{N} 4-\mathrm{N} 5$ & $119.36(16)$ \\
\hline $\mathrm{N} 1-\mathrm{N} 2-\mathrm{C} 9$ & $116.20(16)$ & $\mathrm{N} 4-\mathrm{N} 5-\mathrm{C} 23$ & $116.86(15)$ \\
\hline $\mathrm{N} 1-\mathrm{N} 2-\mathrm{C} 8$ & $121.72(16)$ & $\mathrm{N} 4-\mathrm{N} 5-\mathrm{C} 22$ & $121.80(16)$ \\
\hline $\mathrm{C} 9-\mathrm{N} 2-\mathrm{C} 8$ & $121.84(16)$ & $\mathrm{C} 23-\mathrm{N} 5-\mathrm{C} 22$ & $121.17(17)$ \\
\hline $\mathrm{O} 2-\mathrm{N} 3-\mathrm{O} 3$ & $123.55(17)$ & $\mathrm{O} 5-\mathrm{N} 6-\mathrm{O} 6$ & $123.34(18)$ \\
\hline $\mathrm{O} 2-\mathrm{N} 3-\mathrm{C} 4$ & $118.83(17)$ & $\mathrm{O} 5-\mathrm{N} 6-\mathrm{C} 18$ & $118.89(16)$ \\
\hline $\mathrm{O} 3-\mathrm{N} 3-\mathrm{C} 4$ & $117.62(18)$ & $\mathrm{O} 6-\mathrm{N} 6-\mathrm{C} 18$ & $117.76(17)$ \\
\hline $\mathrm{C} 14-\mathrm{S} 1-\mathrm{C} 11$ & $92.35(11)$ & $\mathrm{C} 28-\mathrm{S} 2-\mathrm{C} 25$ & $94.06(11)$ \\
\hline $\mathrm{C} 14-\mathrm{C} 12 \mathrm{~A}-\mathrm{C} 11$ & $92.35(11)$ & $\mathrm{C} 28-\mathrm{C} 26 \mathrm{~A}-\mathrm{C} 25$ & $94.06(11)$ \\
\hline $\mathrm{C} 14-\mathrm{C} 12 \mathrm{~A}-\mathrm{H} 12 \mathrm{~A}$ & 133.8 & $\mathrm{C} 28-\mathrm{C} 26 \mathrm{~A}-\mathrm{H} 26 \mathrm{~A}$ & 133.0 \\
\hline $\mathrm{C} 11-\mathrm{C} 12 \mathrm{~A}-\mathrm{H} 12 \mathrm{~A}$ & 133.8 & $\mathrm{C} 25-\mathrm{C} 26 \mathrm{~A}-\mathrm{H} 26 \mathrm{~A}$ & 133.0 \\
\hline $\mathrm{C} 6-\mathrm{C} 1-\mathrm{C} 2-\mathrm{C} 3$ & $0.1(3)$ & $\mathrm{C} 20-\mathrm{C} 15-\mathrm{C} 16-\mathrm{C} 17$ & $-1.7(3)$ \\
\hline $\mathrm{C} 7-\mathrm{C} 1-\mathrm{C} 2-\mathrm{C} 3$ & $178.96(18)$ & $\mathrm{C} 21-\mathrm{C} 15-\mathrm{C} 16-\mathrm{C} 17$ & $178.39(16)$ \\
\hline $\mathrm{C} 1-\mathrm{C} 2-\mathrm{C} 3-\mathrm{C} 4$ & $-0.1(3)$ & $\mathrm{C} 15-\mathrm{C} 16-\mathrm{C} 17-\mathrm{C} 18$ & $0.4(3)$ \\
\hline $\mathrm{C} 2-\mathrm{C} 3-\mathrm{C} 4-\mathrm{C} 5$ & $0.2(3)$ & $\mathrm{C} 16-\mathrm{C} 17-\mathrm{C} 18-\mathrm{C} 19$ & $0.6(3)$ \\
\hline $\mathrm{C} 2-\mathrm{C} 3-\mathrm{C} 4-\mathrm{N} 3$ & $-179.27(18)$ & $\mathrm{C} 16-\mathrm{C} 17-\mathrm{C} 18-\mathrm{N} 6$ & $-179.94(16)$ \\
\hline $\mathrm{C} 3-\mathrm{C} 4-\mathrm{C} 5-\mathrm{C} 6$ & $-0.3(3)$ & $\mathrm{C} 17-\mathrm{C} 18-\mathrm{C} 19-\mathrm{C} 20$ & $-0.1(3)$ \\
\hline $\mathrm{N} 3-\mathrm{C} 4-\mathrm{C} 5-\mathrm{C} 6$ & $179.15(17)$ & $\mathrm{N} 6-\mathrm{C} 18-\mathrm{C} 19-\mathrm{C} 20$ & $-179.61(17)$ \\
\hline $\mathrm{C} 4-\mathrm{C} 5-\mathrm{C} 6-\mathrm{C} 1$ & $0.3(3)$ & $\mathrm{C} 18-\mathrm{C} 19-\mathrm{C} 20-\mathrm{C} 15$ & $-1.3(3)$ \\
\hline
\end{tabular}




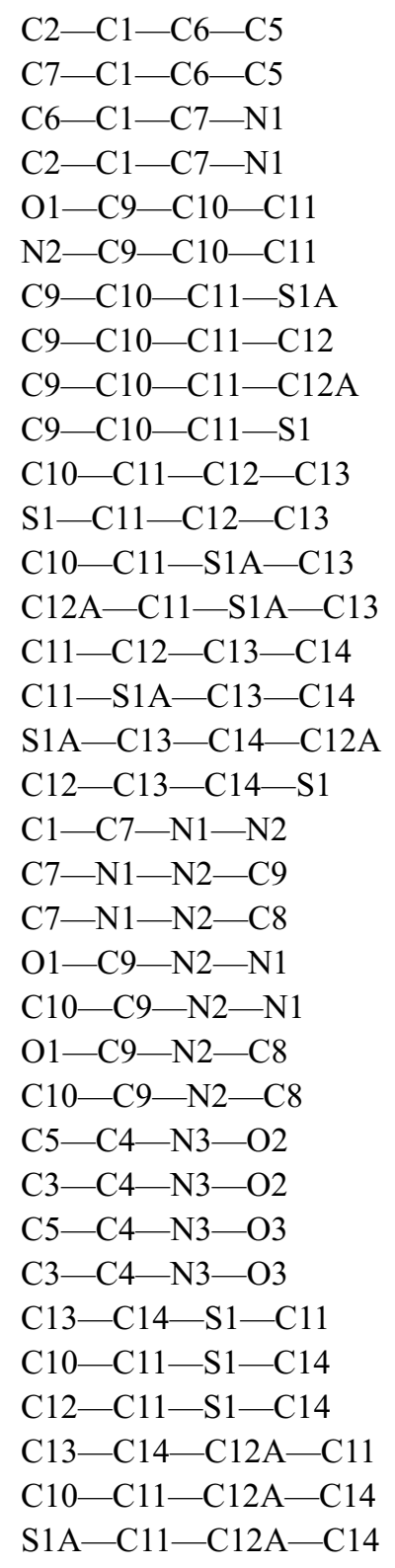

$-0.2(3)$
$-179.11(17)$
$-176.17(17)$
$5.0(3)$
$-87.5(2)$
$91.7(2)$
$118.60(17)$
$118.60(17)$
$-61.7(2)$
$-61.7(2)$
$-179.11(16)$
$1.14(15)$
$-179.11(16)$
$1.14(15)$
$-0.1(2)$
$-0.1(2)$
$-0.9(3)$
$-0.9(3)$
$-177.33(15)$
$-176.72(16)$
$8.8(3)$
$178.83(16)$
$-0.5(2)$
$-6.7(3)$
$174.00(16)$
$-8.9(3)$
$170.54(18)$
$171.05(19)$
$-9.5(3)$
$1.36(18)$
$178.76(16)$
$-1.49(14)$
$1.36(18)$
$178.76(16)$
$-1.49(14)$

$2.2(3)$

$-177.92(17)$

$11.1(3)$

$-169.05(17)$

$-8.7(3)$

$171.09(17)$

87.0 (2)

$87.0(2)$

$-94.5(2)$

$-94.5(2)$

$176.91(19)$

$-1.77(19)$

$176.91(19)$

$-1.77(19)$

1.4 (3)

$1.4(3)$

$-0.3(3)$

$-0.3(3)$

$-177.94(15)$

$-178.41(16)$

$6.3(3)$

$-177.20(16)$

$3.0(2)$

$-1.9(3)$

178.24 (17)

$-8.7(3)$

$170.78(17)$

$171.80(17)$

$-8.7(3)$

$-0.6(2)$

$1.42(16)$

$-177.37(18)$

$-0.6(2)$

$1.42(16)$

$-177.37(18)$

Hydrogen-bond geometry $\left(\AA,{ }^{\circ}\right)$

$\mathrm{Cg} 6$ is the centroid of the $\mathrm{C} 15-\mathrm{C} 20$ ring.

\begin{tabular}{lllll}
\hline$D-\mathrm{H} \cdots A$ & $D-\mathrm{H}$ & $\mathrm{H} \cdots A$ & $D \cdots A$ & $D-\mathrm{H} \cdots A$ \\
\hline $\mathrm{C} 5-\mathrm{H} 5 \cdots \mathrm{O} 2^{\mathrm{i}}$ & 0.95 & 2.48 & $3.312(3)$ & 147 \\
$\mathrm{C} 6-\mathrm{H} 6 \cdots 6^{\mathrm{ii}}$ & 0.95 & 2.56 & $3.412(2)$ & 149 \\
$\mathrm{C} 7-\mathrm{H} 7 \cdots \mathrm{O}^{\mathrm{ii}}$ & 0.95 & 2.41 & $3.281(3)$ & 153 \\
$\mathrm{C} 14-\mathrm{H} 14 \cdots \mathrm{O} 4$ & 0.95 & 2.55 & $3.464(3)$ & 160 \\
$\mathrm{C} 17-\mathrm{H} 17 \cdots{ }^{\mathrm{iii}}$ & 0.95 & 2.43 & $3.104(2)$ & 128 \\
$\mathrm{C} 20-\mathrm{H} 20 \cdots{ }^{\mathrm{iii}}$ & 0.95 & 2.33 & $3.176(2)$ & 147
\end{tabular}


supporting information

$\begin{array}{lllll}\mathrm{C} 8-\mathrm{H} 8 B \cdots C g 6^{\mathrm{v}} & 0.98 & 2.77 & 3.634(2) & 147 \\ \mathrm{C} 24-\mathrm{H} 24 A \cdots C g 6^{\mathrm{vi}} & 0.98 & 2.77 & 3.628(2) & 145\end{array}$

Symmetry codes: (i) $-x+2,-y,-z$; (ii) $x+1, y, z-1$; (iii) $-x-1,-y+1,-z+1$; (iv) $-x+1,-y,-z+1$; (v) $-x,-y+1,-z+1$; (vi) $x+1, y, z$. 